Electronic Journal of Statistics

Vol. 14 (2020) 1707-1743

ISSN: $1935-7524$

https://doi.org/10.1214/20-EJS1705

\title{
Posterior contraction and credible sets for filaments of regression functions
}

\author{
Wei Li \\ Department of Mathematics \\ Syracuse University \\ Syracuse, New York, 13244 \\ e-mail: wli169@syr.edu \\ Subhashis Ghosal \\ Department of Statistics \\ North Carolina State University \\ Raleigh, North Carolina, 27695 \\ e-mail: sghosal@stat.ncsu.edu
}

\begin{abstract}
A filament consists of local maximizers of a smooth function $f$ when moving in a certain direction. A filamentary structure is an important feature of the shape of an object and is also considered as an important lower dimensional characterization of multivariate data. There have been some recent theoretical studies of filaments in the nonparametric kernel density estimation context. This paper supplements the current literature in two ways. First, we provide a Bayesian approach to the filament estimation in regression context and study the posterior contraction rates using a finite random series of B-splines basis. Compared with the kernel-estimation method, this has a theoretical advantage as the bias can be better controlled when the function is smoother, which allows obtaining better rates. Assuming that $f: \mathbb{R}^{2} \mapsto \mathbb{R}$ belongs to an isotropic Hölder class of order $\alpha \geq 4$, with the optimal choice of smoothing parameters, the posterior contraction rates for the filament points on some appropriately defined integral curves and for the Hausdorff distance of the filament are both $(n / \log n)^{(2-\alpha) /(2(1+\alpha))}$. Secondly, we provide a way to construct a credible set with sufficient frequentist coverage for the filaments. We demonstrate the success of our proposed method in simulations and one application to earthquake data.
\end{abstract}

MSC 2010 subject classifications: Primary 62G08, 62G20; secondary $62 \mathrm{G} 15$.

Keywords and phrases: Filament, nonparametric regression, posterior contraction, credibility, coverage, B-splines.

Received June 2019.

\section{Contents}

1 Introduction . . . . . . . . . . . . . . . . . . . . . 1708

2 Notations and preliminaries . . . . . . . . . . . . . . . . . 1710

3 Model, prior and posterior . . . . . . . . . . . . . . . 1712

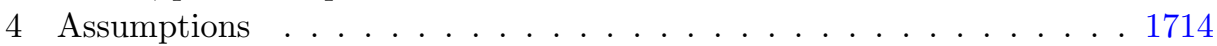


5 Posterior contraction and credible sets for filaments . . . . . . . 1715

6 Simulation . . . . . . . . . . . . . . . . . . . . . . . . 1719

7 Application . . . . . . . . . . . . . . . . . . . . . . . 1722

A Technical proofs . . . . . . . . . . . . . . . . . . . 1723

A.1 Some lemmas . . . . . . . . . . . . . . . . . . . . . . 1724

A.2 Proofs of the main results . . . . . . . . . . . . . 1727

References . . . . . . . . . . . . . . . . . . . . . . . . . 1741

\section{Introduction}

There is a large body of literature on the problem of estimating intrinsic lower dimensional structure of multivariate data. A filament or a ridgeline is one of such geometric objects that draws a lot of attention in the recent years. Intuitively speaking, a filament consists of local maximizers of a smooth function $f$ (say a density or a regression function) when moving in a certain direction. Roughly speaking these are generalized modes that reside on hyperplanes that are normal to the steepest ascent direction.

The filamentary structure (ridges) together with the valleys (i.e. local minimizers counterparts of ridges), critical points are the main features of the shapes of objects. They are common in medical images, satellite images and many three dimensional objects. One important example comes from the study of the cosmic web - a large scale web structure of galaxies (clusters) connected by long threads composed of sparse hydrogen gas. These intergalactic connections are believed to trace the filaments of dark matters. The discovery and study of the dark matter is a key challenge of cosmology. For more references, see Novikov et al. [28], Dietrich et al. [14] and Chen et al. [7].

The filament estimation falls into a broad category of data analytic methods that are called topological data analysis which is used to find intrinsic structure in data (Wasserman [37]). In particular, it is closely related to manifold learning problem. Manifold learning problem assumes that the data points are generated from some a priori unknown lower dimensional structure with background noises. Arias-Castro et al. [1] developed a test to detect if a dataset contains some small fraction of data points that are supported on a curve. Genovese et al. [17] and Genovese et al. [18] studied the problem of estimation of the manifold. They showed that the ridge of a density function can serve as a surrogate to the manifold and can be estimated with a better rate. Since the filaments can be considered as generalized modes, relevant literature includes those on mode (and maximum) estimation, for instance, Shoung and Zhang [33], Facer and Müller [16] and a recent paper by Yoo and Ghosal [39] in the Bayesian framework.

The statistical properties of the estimated filaments, like convergence rates and limiting distribution, have been studied in a few recent papers. Genovese et al. [18] established the convergence rates of the filament obtained from the kernel density estimation. Chen, Genovese and Wasserman [6] provided BerryEsseen type results for the limiting distribution. With a different approach, 
Qiao and Polonik [30] also established the convergence rates and the extreme value type results for limiting distribution. Constructing confidence bands in nonparametric problems is also a very important topic in itself. Some recent papers include Claeskens and Van Keilegom [12], Giné and Nickl [19] and Chernozhukov et al. $[8,9]$. But these works focus primarily on the standard functions, e.g., regressions and density functions. It is worth to point out that in the problems of filaments, the estimation and inference is performed for a set, or more broadly speaking for some geometric object (Molchanov [27]). A similar example is the level set of a certain function Jankowski and Stanberry [21], Mason and Polonik [26], Mammen and Polonik [25]. Some other examples arise in econometrics literature where partially identified parameters (as a set) is the object of interest; see Chernozhukov et al. [10,11] and references therein. Very little is known about how to make inference about a geometric target in general and this remains a very important area of study. For the problems of filaments, Chen, Genovese and Wasserman [6] developed a bootstrap-based method for uncertainty quantification.

Frequentist properties of Bayesian procedure for an unknown function has also been an area receiving much attention. In particular, posterior contraction rates for functions in different $L_{r}$-metrics have been studied in Giné and Nickl [20], Castillo [5] and Yoo and Ghosal [38]. There also have been many studies on constructing credible regions which have the right frequentist coverage. Szabó et al. [34] studied adaptive $L_{2}$-credible regions in Gaussian white noise model. Yoo and Ghosal [38] constructed $L_{\infty}$-credible regions in the multivariate nonparametric regression setting with known smoothness condition. Knapik et al. [23] studied the frequentist coverage of credible sets in nonparametric inverse problems. Belitser [2] studied credible sets in mildly ill-posed inverse signal-inwhite-noise model. Belitser and Nurushev [4] studied uncertainty quantification for the unknown, possibly sparse, signal in general signal with noise models. van der Pas et al. [35] studied credible sets using the horseshoe prior in the sparse multivariate normal means model in an adaptive setting. Ray [31] studied Bernstein-von Mises theorems for adaptive nonparametric Bayesian procedures in the Gaussian white noise model. Yoo and Ghosal [39] studied Bayesian mode and maximum estimation and provided credible sets with good coverage. Belitser and Ghosal [3] studied uncertainty quantification for high dimensional linear regression models and their results are also extended to high dimensional additive nonparametric regression models. Using credible regions with sufficiently frequentist coverage, one can obtain confidence regions for the truth in the frequentist sense relatively easily from the posterior distribution. This is especially appealing when the object to be studied is complicated.

So far in the literature, the study of filaments is limited only to densities only using the kernel approach. This paper supplements the current literature in two ways. First, we provide a Bayesian approach to the filament estimation in regression context and study the contraction rates using a finite random series of B-splines basis. This has theoretical advantages as the bias can be better controlled when the function is more than "minimally smooth" (i.e. more than four times differentiable). Specifically, better rates are obtained for the filament 
points on some appropriately defined integral curves and for the Hausdorff distance of the filament which are both $(n / \log n)^{(2-\alpha) /(2(1+\alpha))}$ (see Remarks 5.3 and 5.4). Secondly, we provide a way to construct credible set with sufficient frequentist coverage for the filaments (see Theorem 5.9). In contrast to the bootstrap-based confidence region proposed by Chen, Genovese and Wasserman [6] which gives a band-shape region, our valid credible region consists of filaments from posterior samples. Another difference is that the inferential target in this paper is the true quantity itself, while in Chen, Genovese and Wasserman [6] the inference is directed towards to the debiased quantity. Although relying on some existing results in the literature, these additional results we obtain offer a new perspective how a Bayesian approach can be applied to a complicated nonparametric problem in a rather intuitive way.

Before we move on to the formal definition of the filament, it is worth to point out that some other possible definitions of filaments have also been discussed and studied in mathematics and computer sciences literature; see Eberly [15] for more details. In this paper, we study the filament as introduced in Chen, Genovese and Wasserman [6] and Qiao and Polonik [30].

This paper is organized as follows. Notation and background materials are given in Section 2. The model is formally introduced in Section 3 along with the descriptions of the prior distribution and the posterior distribution. Technical assumptions are given in Section 4. The main results in posterior contraction and credible region are presented in Section 5. Simulation results and an application to an earthquake data are presented in Section 6 and 7 respectively. All proofs are given in Section A.

\section{Notations and preliminaries}

Let $\mathbb{N}=\{1,2, . .\},, \mathbb{N}_{0}=\{0,1,2,3, \ldots\}$. Given two real sequences $a_{n}$ and $b_{n}$, $a_{n}=O\left(b_{n}\right)$ or $a_{n} \lesssim b_{n}$ means that $a_{n} / b_{n}$ is bounded, while $a_{n}=o\left(b_{n}\right)$ or $a_{n} \ll b_{n}$ means that both $a_{n} / b_{n} \rightarrow 0$. Also $a_{n} \asymp b_{n}$ means that $a_{n}=O\left(b_{n}\right)$ and $b_{n}=O\left(a_{n}\right)$. For a sequence of random elements $Z_{n}, Z_{n}=O_{p}\left(a_{n}\right)$ means that $\mathrm{P}\left(\left|Z_{n}\right| \leq C a_{n}\right) \rightarrow 1$ for some constant $C>0$.

For a vector $x \in \mathbb{R}^{d}$, we define $\|x\|_{p}=\left(\sum_{k=1}^{d}\left|x_{k}\right|^{p}\right)^{1 / p}$ for $0 \leq p<\infty$, $\|x\|_{\infty}=\max _{1 \leq k \leq d}\left|x_{k}\right|$ and write $\|x\|$ for $\|x\|_{2}$. The scalar product of two vectors $x$ and $y$ will be written as $x^{T} y$ or $\langle x, y\rangle$. For an $m \times m$ matrix $A$, let $\|A\|_{(2,2)}=\left(\lambda_{\max }\left(A^{T} A\right)\right)^{1 / 2}$, where $\lambda_{\max }$ denotes the largest eigenvalue; $\|A\|_{(\infty, \infty)}=\max _{1 \leq i \leq m} \sum_{j=1}^{m}\left|a_{i j}\right|$ and $\|A\|_{F}=\sqrt{\operatorname{tr}\left(A^{T} A\right)}$ the Frobenius norm of matrix $A$. Given another $m \times m$ matrix $B, B \leq A$ means $A-B$ is non-negative definite. We also denote an $n$ by $n$ identity matrix by $I_{n}$.

For $f: U \mapsto \mathbb{R}$ on some bounded set $U \subset \mathbb{R}^{d}$. Let $\|f\|_{p}$ be the $L_{p}$ norm and $\|f\|_{\infty}=\sup _{x \in U}|f(x)|$. For $g: U \mapsto \mathbb{R}$ on some bounded set $U \subset \mathbb{R}^{d}$, let $\nabla g$ be the gradient of $g$, which is a $d \times 1$ vector of functions. For a $d$ dimensional multindex $r=\left(r_{1}, \ldots, r_{d}\right) \in \mathbb{N}_{0}^{d}$, let $D^{r}$ be the partial derivative operator $\partial^{|r|} / \partial x_{1}^{r_{1}} \cdots \partial x_{d}^{r_{d}}$ where $|r|=\sum_{k=1}^{d} r_{k}$. 
The Hölder Space $\mathcal{H}^{\alpha}\left([0,1]^{d}\right)$ of order $\alpha>0$ consists of functions $f:[0,1]^{d} \mapsto$ $\mathbb{R}$ such that $\|f\|_{\alpha, \infty}<\infty$, where $\|\cdot\|_{\alpha, \infty}$ is the Hölder norm

$$
\|f\|_{\alpha, \infty}=\max _{r:|r| \leq\lfloor\alpha\rfloor} \sup _{x}\left|D^{r} f(x)\right|+\max _{r:|r|=\lfloor\alpha\rfloor} \sup _{x, y: x \neq y} \frac{\left|D^{r} f(x)-D^{r} f(y)\right|}{\|x-y\|^{\alpha-\lfloor\alpha\rfloor}},
$$

where sup is taken over the support of $f$ and $\lfloor\alpha\rfloor$ is the largest integer strictly smaller than $\alpha$.

The filament or the ridge line of a smooth function defined on $\mathbb{R}^{2}$ is a collection of points at which the gradient of the function is orthogonal to the eigenvector of its Hessian that corresponds to the most negative eigenvalue (if exists). The filament point (the point on the filament) is a generalization of mode of the function. To see this connection, recall a well-known result that tests for local maximum point (mode).

Let $f: \mathbb{R}^{2} \mapsto \mathbb{R}$ be a smooth function, $\nabla f=\left(f^{(1,0)}, f^{(0,1)}\right)^{T}$ be the gradient and $H f$ be the Hessian. Recall a test for a local maximum point is the following

$$
a^{T} \nabla f(x)=0, \quad a^{T} H f(x) a<0,
$$

for all nonzero vector $a$. Let $V(x)$ be the eigenvector of $H f(x)$ that corresponds to the smallest eigenvalues $\lambda(x)$. A point $x \in \mathbb{R}^{2}$ is called a filament point or ridge point if

$$
V^{T}(x) \nabla f(x)=0, \quad V^{T}(x) H f(x) V(x)<0 .
$$

Therefore, a filament point is a point at which the function has a local maximum along the direction given by $V$. Note also that $V^{T}(x) H f(x) V(x)<0$ is equivalent to $\lambda(x)<0$.

More generally, for $f$ defined on $\mathbb{R}^{d}, 0 \leq s \leq d-1$, the eigenvectors of $H f(x)$ can be used to define two orthogonal spaces, namely, a $(d-s)$-dimensional normal space (corresponding to $(d-s)$-eigenvectors with smallest eigenvalues) and an $s$-dimensional tangent space (corresponding to the remaining eigenvectors). An $s$-dimensional filament point on $\mathbb{R}^{d}$ is a point where the gradient of $f$ is orthogonal to the normal space and the eigenvalues associated with the normal space are all (strongly) negative. Alternatively, such a point $x$ can be regarded as a point where $f$ attains the local maximum in the affine space spanned by the normal space translated by $x$. The modes are then simply 0-dimensional filaments. The 1-dimensional filament on $\mathbb{R}^{2}$ is of primary interest in our discussion here.

From now on, $f$ is assumed to be some smooth bivariate regression function. Suppose that the Hessian matrix $H f(x)$ of $f$ at $x$ has eigvenvector $V(x)$ corresponding to the smaller eigenvalue $\lambda(x)$. The filament $\mathcal{L}(f)$ of the regression function $f$ is formally defined as

$$
\mathcal{L}=\mathcal{L}(f):=\{x:\langle\nabla f(x), V(x)\rangle=0 \text { and } \lambda(x)<0\} .
$$

We also introduce an integral curve, which is the solution to the following differential equation

$$
\frac{d \Upsilon_{x_{0}}(t)}{d t}=V\left(\Upsilon_{x_{0}}(t)\right), \quad \Upsilon_{x_{0}}(0)=x_{0}
$$


where $x_{0}$ is some starting point from a sufficiently rich set $\mathcal{G}$ to be described in Section 4 . We define the "hitting time" of the filament by traversing the integral curve starting at a point $x_{0}$,

$$
t_{x_{0}}=\underset{t}{\operatorname{argmin}}\left\{|t| \geq 0:\left\langle\nabla f\left(\Upsilon_{x_{0}}(t)\right), V\left(\Upsilon_{x_{0}}(t)\right)\right\rangle=0, \lambda\left(\Upsilon_{x_{0}}(t)\right)<0\right\} .
$$

The integral curves will be our intermediate object for the study of the filament (as a collection of points on these curves). It is obvious that $\Upsilon_{x_{0}}\left(t_{x_{0}}\right) \in \mathcal{L}$. Taking a plug-in estimation's point of view, $\Upsilon_{x_{0}}$ and $t_{x_{0}}$ can be estimated, and therefore a filament point (on certain integral curve) can be estimated. Through the pointwise comparison between the estimated filament point and the true filament point over a large collection of starting points, one can assess the performance of the estimation procedure. This idea is put forward in Qiao and Polonik [30] and is useful for our study.

\section{Model, prior and posterior}

Throughout the paper, let $d=2$, thus $x=\left(x_{1}, x_{2}\right)$. We consider the nonparametric regression model,

$$
Y_{i}=f\left(X_{i}\right)+\varepsilon_{i},
$$

where $\varepsilon_{i}$ are independent and identically distributed (i.i.d) Gaussian random variables with mean 0 and variance $\sigma^{2}$ for $i=1, \ldots, n$. Without loss of generality, we let $X_{i}$ takes values in $[0,1]^{2}$. Let $Y=\left(Y_{1}, \ldots, Y_{n}\right)^{T}, X=\left(X_{1}^{T}, \ldots, X_{n}^{T}\right)^{T}$, $F=\left(f\left(X_{1}\right), \ldots, f\left(X_{n}\right)\right)^{T}$ and $\varepsilon=\left(\varepsilon_{1}, \ldots, \varepsilon_{n}\right)^{T}$, then we can write $Y=F+\varepsilon$.

To model $f$, we shall use B-spline function as our basis function. Given some $q \in \mathbb{N}, N \in \mathbb{N}$, for a sequence of knots $0=t_{-(q-1)}=\cdots=t_{0}<\cdots<$ $t_{N+1}=\cdots=t_{N+q}=1$, denote the univariate B-spline function of order $q$ by $B_{i, q}(x)=\left(t_{i}-t_{i-q}\right)\left[t_{i-q}, \cdots, t_{i}\right](\cdot-x)_{+}^{q-1}, i=1,2, \ldots, N+q$ (De Boor [13]). Here $\left[t_{i-q}, \cdots, t_{i}\right](\cdot-x)_{+}^{q-1}$ is the divided difference of the function $y \mapsto(y-x)_{+}^{q-1}$, where $(y-x)_{+}^{0}=\mathbb{1}(y \geq x),(y-x)_{+}^{q-1}=(y-x)^{q-1} \mathbb{1}(y \geq x)$. By construction, $B_{i, q}(x)>0$ on $\left(t_{i-q}, t_{i}\right)$ and $\sum_{i=1}^{N+q} B_{i, q}(x)=1$. Now to construct a basis on $\mathbb{R}^{2}$, for $x=\left(x_{1}, x_{2}\right) \in \mathbb{R}^{2}$, define $b_{J_{1}, J_{2}, q_{1}, q_{2}}(x)=\left(B_{j_{1}, q_{1}}\left(x_{1}\right) B_{j_{2}, q_{2}}\left(x_{2}\right): 1 \leq\right.$ $\left.j_{k} \leq J_{k}, k=1,2\right)^{T}$ to be a vector of tensor product of B-splines functions, with possibly different orders $q_{k}$ and knot sequences in different directions, i.e, $0=t_{k,-\left(q_{k}-1\right)}=\cdots=t_{k, 0}<t_{k, 1}<\ldots<t_{k, N_{k}}<t_{k, N_{k}+1}=\cdots=t_{k, N_{k}+q_{k}}=1$ for $k=1,2$. Here $N_{k}$ denotes the number of interior points and $J_{k}=q_{k}+N_{k}$ denotes the number of basis functions on the $\mathrm{k}$-th coordinate. The elements of this vector is assumed to be in dictionary order according to their indices. For each $k=1,2$, define $\delta_{k, \ell}=t_{k, \ell+1}-t_{k, \ell}$ for $\ell=0, \ldots, N_{k}$ and assume that $\max _{1 \leq \ell \leq N_{k}} \delta_{k, \ell} / \min _{1 \leq \ell \leq N_{k}} \delta_{k, \ell} \leq C$ for some $C>0$. This assumption is clearly satisfied for the uniform partition. Whenever $q_{1}, q_{2}$ are considered fixed, we shall suppress the subscripts $q_{1}, q_{2}$ in our notations of B-spline functions, for instance we write $b_{J_{1}, J_{2}}$ for $b_{J_{1}, J_{2}, q_{1}, q_{2}}$. Following a similar set-up in Yoo and Ghosal [38], we first put a random tensor-product $\mathrm{B}$ series prior on $f$. Let $q_{1}, q_{2}$ fixed and 
$N_{k}=N_{k}(n)$ and hence $J_{k}=J_{k}(n)$. Letting $f\left(X_{i}\right)=b_{J_{1}, J_{2}}^{T}\left(X_{i}\right) \theta$ for some vector $\theta \in \mathbb{R}^{J_{1} J_{2}}$, our model becomes

$$
Y \mid\left(X, \theta, \sigma^{2}\right) \sim \mathrm{N}\left(B \theta, \sigma^{2} I_{n}\right),
$$

where $B=\left(b_{J_{1}, J_{2}}\left(X_{1}\right), \ldots, b_{J_{1}, J_{2}}\left(X_{n}\right)\right)^{T}$.

Throughout we will use superscript * to denote the true values. Even though our model for $Y$ is Gaussian, the true error terms are only required to be subGaussian. Specifically, we assume the data are i.i.d from some true distribution $\mathrm{P}_{0}$ where $Y_{i}=f^{*}\left(X_{i}\right)+\varepsilon_{i}$ with i.i.d. sub-Gaussian $\varepsilon_{i}$ whose mean is 0 and variance $\sigma_{0}^{2}$ for $i=1, \ldots, n$. Our study allows both fixed and random design cases. If $\left\{X_{i}: i=1, \ldots, n\right\}$ are considered fixed data points, we assume that for some cumulative distribution function $\mathbb{G}$ with positive and continuous density

$$
\sup _{x \in[0,1]^{2}}\left|\mathbb{G}_{n}(x)-\mathbb{G}(x)\right|=o\left(\frac{1}{J_{1} J_{2}}\right),
$$

where $\mathbb{G}_{n}$ is the empirical distribution of $\left\{X_{i}: i=1, \ldots, n\right\}$. If $X_{i} \stackrel{\text { i.i.d. }}{\sim} \mathbb{G}$, then the above condition is satisfied with probability tending to one as long as $J_{1} \asymp J_{2} \asymp o\left(n^{1 / 4}\right)$ by Donsker's theorem. In both cases, we shall use $\mathbb{D}_{n}$ to denote all observations.

We assign $\theta \mid \sigma^{2} \sim \mathrm{N}\left(\theta_{0}, \sigma^{2} \Lambda_{0}\right)$ for some $\theta_{0} \in \mathbb{R}^{J_{1} J_{2}}$, assuming that for some constants $0<c_{1} \leq c_{2}<\infty$ it holds that

$$
c_{1} I_{J_{1} J_{2}} \leq \Lambda_{0} \leq c_{2} I_{J_{1} J_{2}},
$$

where $I_{J_{1} J_{2}}$ is a $J_{1} J_{2}$ by $J_{1} J_{2}$ identity matrix. It follows then

$$
\theta \mid \mathbb{D}_{n}, \sigma^{2} \sim \mathrm{N}\left(\left(\Lambda_{0}^{-1}+B^{T} B\right)^{-1}\left(B^{T} Y+\Lambda_{0}^{-1} \theta_{0}\right), \sigma^{2}\left(B^{T} B+\Lambda_{0}^{-1}\right)^{-1}\right) .
$$

The posterior distribution for $f(x)$ and its partial derivatives are then obtained accordingly. Define the vector

$$
b_{J_{1}, J_{2}}^{(r)}(x)=\left(\frac{\partial^{r_{1}}}{\partial x_{1}^{r_{1}}} B_{j_{1}, q_{1}}\left(x_{1}\right) \frac{\partial^{r_{2}}}{\partial x_{2}^{r_{2}}} B_{j_{2}, q_{2}}\left(x_{2}\right): 1 \leq j_{k} \leq J_{k}, k=1,2\right)^{T} .
$$

Therefore,

$$
\begin{aligned}
& \Pi\left(D^{r} f \mid \mathbb{D}_{n}, \sigma^{2}\right) \sim \operatorname{GP}\left(A_{r} Y+C_{r} \theta_{0}, \sigma^{2} \Sigma_{r}\right), \\
& A_{r}(x)=b_{J_{1}, J_{2}}^{(r)}(x)^{T}\left(B^{T} B+\Lambda_{0}^{-1}\right)^{-1} B^{T}, \\
& C_{r}(x)=b_{J_{1}, J_{2}}^{(r)}(x)^{T}\left(B^{T} B+\Lambda_{0}^{-1}\right)^{-1} \Lambda_{0}^{-1}, \\
& \Sigma_{r}(x, y)=b_{J_{1}, J_{2}}^{(r)}(x)^{T}\left(B^{T} B+\Lambda_{0}^{-1}\right)^{-1} b_{J_{1}, J_{2}}^{(r)}(y),
\end{aligned}
$$

where GP denotes a Gaussian process. Notice that under $\mathrm{P}_{0}, A_{r}(x) \varepsilon / \sigma_{0}$ is a mean-zero process with a sub-Gaussian tail. 
To handle $\sigma^{2}$, we can either put a conjugate inverse-gamma prior $\sigma^{2} \sim$ $\operatorname{IG}(a / 2, b / 2)$ with shape parameter $a / 2>2$ and rate parameter $b / 2>0$ or plug-in an estimate for $\sigma^{2}$. Since the theory does not make much difference, for ease of exposition, we shall use the second approach, to be called the empirical Bayes method. The empirical Bayes has the following posterior distribution (Yoo and Ghosal [38])

$$
\Pi\left(D^{r} f \mid \mathbb{D}_{n}\right) \sim \operatorname{GP}\left(A_{r} Y+C_{r} \theta_{0}, \hat{\sigma}^{2} \Sigma_{r}\right),
$$

where

$$
\hat{\sigma}^{2}=n^{-1}\left(Y-B \theta_{0}\right)^{T}\left(B \Lambda_{0} B^{T}+I_{n}\right)^{-1}\left(Y-B \theta_{0}\right)
$$

\section{Assumptions}

We follow the standard assumptions in Qiao and Polonik [30]. For convenience, we let $d^{2} f(x)=\left(f^{(2,0)}(x), f^{(1,1)}(x), f^{(0,2)}(x)\right)^{T}$ and sometimes write $D^{r} f=$ $f^{(r)}$. We assume that the two eigenvalues of $H f(x)$ are distinct. Then $V(x)$ and $\lambda(x)$ take the following forms $V(x)=G\left(d^{2} f(x)\right)$ and $\lambda(x)=J\left(d^{2} f(x)\right)$ for some function $G=\left(G_{1}, G_{2}\right)^{T}: \mathbb{R}^{3} \rightarrow \mathbb{R}^{2}$ and $J: \mathbb{R}^{3} \rightarrow \mathbb{R}$ given by

$$
\begin{aligned}
& G(u, v, w)=\left(\begin{array}{c}
2 u-2 w+2 v-2 \sqrt{(w-u)^{2}+4 v^{2}} \\
w-u+4 v-\sqrt{(w-u)^{2}+4 v^{2}},
\end{array}\right), \\
& J(u, v, w)=\frac{1}{2}\left(u+w-\sqrt{(u-w)^{2}+4 v^{2}}\right) .
\end{aligned}
$$

Throughout the proofs, we may take the normalized version of the eigenvector $V$, that is, $\|V\|=1$. This is not necessary but it simplifies discussion.

For some $a^{*}>0$, define $\mathcal{G}=\left\{\Upsilon_{x_{0}}^{*}(t): x_{0} \in \mathcal{L}^{*},-a^{*} \leq t \leq a^{*}\right\}$. We choose $a^{*}$ small so that $\mathcal{G} \subset[0,1]^{2}$ and $t_{x_{0}}^{*}$ is unique for any $x_{0} \in \mathcal{G}$. Define $\mathcal{L}^{*} \oplus \delta=$ $\cup_{x \in \mathcal{L}^{*}} B(x, \delta)$, where $B(x, \delta)$ is an open ball around $x$ of radius $\delta$. The following assumptions will be needed for the theory.

(A1) The truth $f^{*}$ belongs to a Hölder Space $\mathcal{H}^{\alpha}\left([0,1]^{2}\right)$ with $\alpha \geq 4$.

(A2) There is some $\delta>0$ small, such that for all $x \in\left(\mathcal{L}^{*} \oplus \delta\right) \cap[0,1]^{2}$, $H f^{*}(x)$ has two distinct eigenvalues, with smaller eigenvalue $\lambda^{*}(x) \leq-\eta$ for some small positive value $\eta$.

(A3) For the $\delta>0$ in (A2), for all $x \in \mathcal{L}^{*} \oplus \delta,\left|\left\langle\nabla\left\langle\nabla f^{*}(x), V^{*}(x)\right\rangle, V^{*}(x)\right\rangle\right| \geq$ $\eta$ for the same positive value $\eta$ in (A2).

(A4) The filament $\mathcal{L}^{*}$ is a compact set such that $\mathcal{L}^{*}=\left\{\Upsilon_{x_{0}}^{*}\left(t_{x_{0}}^{*}\right): x_{0} \in \mathcal{G}\right\}$.

(A5) Assume that there exits some $C_{\mathcal{G}}>0$, for any $x_{0} \in \mathcal{G}$,

$$
\inf _{x_{0} \in \mathcal{G} t_{x_{0}}^{*}-a^{*} \leq s<u \leq t_{x_{0}}^{*}+a^{*}} \inf \left\|\frac{\Upsilon_{x_{0}}^{*}(u)-\Upsilon_{x_{0}}^{*}(s)}{u-s}\right\| \geq C_{\mathcal{G}} .
$$


Assumption (A2) is important for our analysis as it guarantees the smoothness of $V^{*}$ (ref. (3.1) of Qiao and Polonik [30]) and that the normal vector of the filament $\nabla\left\langle\nabla f^{*}(x), V^{*}(x)\right\rangle$ is well-defined. Similar assumptions are needed in Genovese et al. [18] and Chen, Genovese and Wasserman [6].

Assumption (A3) says that the normal vector of the filament $\nabla\left\langle\nabla f^{*}(x)\right.$, $\left.V^{*}(x)\right\rangle$ is not orthogonal to $V^{*}(x)$. In addition, it implies that $\nabla\left\langle\nabla f^{*}(x)\right.$, $\left.V^{*}(x)\right\rangle \neq 0$, i.e, the set $\left\{x:\left\langle\nabla f^{*}(x), V^{*}(x)\right\rangle=0\right\}$ is not "thick". This means that a small change of $x \in \mathcal{L}^{*}$ necessarily changes the sign of $\left\langle\nabla f^{*}(x), V^{*}(x)\right\rangle$. If one restricts attention to the locus defined by $\left\langle\nabla f^{*}(x), V^{*}(x)\right\rangle=0$, noting $\operatorname{rank}\left(\nabla\left\langle\nabla f^{*}(x), V^{*}(x)\right\rangle\right)=1$, the implicit function theorem says that $\mathcal{L}$ is a one-dimensional manifold in $\mathbb{R}^{2}$. If for $x \in \mathcal{L}^{*}, \nabla f^{*}(x)=0$, Assumption (A3) should be interpreted as $\left|\left(V^{*}(x)\right)^{T} H f^{*}(x) V^{*}(x)\right| \geq \eta$ which is then implied by Assumption (A2). To see this, notice that

$$
\left\langle\nabla\left\langle\nabla f^{*}(x), V^{*}(x)\right\rangle, V^{*}(x)\right\rangle=\nabla f^{*}(x)^{T} \nabla V^{*}(x) V^{*}(x)+V^{*}(x)^{T} H f^{*}(x) V^{*}(x),
$$

but $V^{*}(x)^{T} H f^{*}(x) V^{*}(x)=\lambda^{*}(x)$. Assumption (A3) parallels the assumption (A2) in Genovese et al. [18], where they assumed some upper bounds on the quantity related to the third derivative of the density function; see also the assumption (P1) in Chen, Genovese and Wasserman [6]. Assumption (A5) is common in the literature; see Koltchinskii et al. [24], Qiao and Polonik [30]. Several useful consequences of these assumptions are summarized in Remark A.1. It is worth to point out that under these assumptions $H f^{*}(x)$ must admit two distinct eigenvalues over some domain and $V^{*}(x)$ is Lipschitz continuous over this domain.

\section{Posterior contraction and credible sets for filaments}

In this section, we provide the main theoretical results. With a suitable choice of a series prior, the function $f$ from the posterior can induce its own integral curve, filaments and hitting time just as how they are defined previously. The goal is to establish posterior contraction rates of these objects relative to some metrics in a similar spirit of the current literature. Here is a brief outline of these results. Theorem 5.1 provides posterior contraction rates for the integral curve. Proposition 5.2 gives posterior contraction rates for the hitting time. Theorem 5.3 gives the posterior contraction rates for the filament along the integral curve. Theorem 5.8 establishes the posterior contraction rates for the filament around the truth, posterior rates for deviations between the posterior filament and the filament induced by the posterior mean, together with the convergence rates for the filament induced by posterior mean to that of the truth, all in terms of the Hausdorff distance. Theorem 5.9 provides a valid credible set with sufficiently high frequentist coverage.

To simply the presentation, we simply set $J_{1}=J_{2}=J$ for some common positive value $J$ that grows with the sample size $n$. All results remain valid even when $J_{1}$ and $J_{2}$ are not equal but grow at the same rate, which is still denoted by the symbol $J$. 
The following result gives a Bayesian counterpart of Theorem 3.3 of Qiao and Polonik [30]. Our proof is similar to theirs, but a few major technical details are different.

Theorem 5.1. Under Assumptions (A1), (A2) and (A5), for $J_{1}=J_{2}=J \asymp$ $(n / \log n)^{1 /(1+2 \alpha)}$, we have the following posterior contraction rate, for $\epsilon_{n}=$ $(n / \log n)^{(2-\alpha) /(1+2 \alpha)}$ and any $M_{n} \rightarrow \infty$,

$$
\Pi\left(\sup _{x_{0} \in \mathcal{G}} \sup _{t \in\left[t_{x_{0}}^{*}-a^{*}, t_{x_{0}}^{*}+a^{*}\right]}\left\|\Upsilon_{x_{0}}(t)-\Upsilon_{x_{0}}^{*}(t)\right\|>M_{n} \epsilon_{n} \mid \mathbb{D}_{n}\right) \stackrel{\mathrm{P}_{0}}{\longrightarrow} 0 .
$$

Remark 5.1. According to Assumption (H1) and the Theorem 3.3 of Qiao and Polonik [30], their rate is $n^{-2 / 9} \sqrt{\log n}$ assuming that $\alpha=4$. Here with the choice $J \asymp(n / \log n)^{1 /(1+2 \alpha)}$, we obtain a rate which has a better logarithmic factor for the case $\alpha=4$, since then the rate reduces to $(n / \log n)^{-2 / 9}$. Note that if we choose $J \asymp(n / \log n)^{1 /(2(1+\alpha))}$ as it is the optimal choice for estimation of the function $f^{*}$, it is easy to see from the proof that now $\sup _{x} \| d^{2} f(x)-$ $d^{2} f^{*}(x) \|^{2}$ is of order $(n / \log n)^{(2-\alpha) /(1+\alpha)}$ and the term (A.1) in Section A has posterior contraction rate of order $(n / \log n)^{(2-\alpha) /(1+\alpha)}$. Thus the contraction rate will then be $\epsilon_{n}=(n / \log n)^{(2-\alpha) /(2(1+\alpha))}$, which is "suboptimal" in the present context.

The following proposition is a Bayesian analog of Proposition A.1 and Proposition 5.1 of Qiao and Polonik [30]. We can obtain better rates by using different magnitude of the tuning parameter; see the remark after the proposition.

Proposition 5.2. Under Assumptions (A1)-(A5) and let $J_{1}=J_{2}=J \asymp$ $(n / \log n)^{1 /(1+2 \alpha)}$, we have the following posterior contraction rate: for $\epsilon_{n}=$ $(n / \log n)^{(5-2 \alpha) /(2(1+2 \alpha))}$ and any $M_{n} \rightarrow \infty$,

$$
\Pi\left(\sup _{x_{0} \in \mathcal{G}}\left|t_{x_{0}}^{*}-t_{x_{0}}\right|>M_{n} \epsilon_{n} \mid \mathbb{D}_{n}\right) \stackrel{\mathrm{P}_{0}}{\longrightarrow} 0 .
$$

If in addition, $\nabla f^{*}(x)=0$ for all $x \in \mathcal{L}^{*}$, then the rates improve to $\epsilon_{n}=$ $(n / \log n)^{(2-\alpha) /(1+2 \alpha)}$.

Remark 5.2. A better rate can be obtained if we choose $J \asymp(n / \log n)^{1 /(2(1+\alpha))}$. With this choice, by Lemma A.2, $\sup _{x}\left\|\nabla f(x)-\nabla f^{*}(x)\right\|$ has posterior contraction rate $(n / \log n)^{(1-\alpha) /(2(1+\alpha))}$, while $\sup _{x}\left\|V(x)-V^{*}(x)\right\|$ has posterior contraction rate $(n / \log n)^{(2-\alpha) /(2(1+\alpha))}$. Recalling Remark 5.1, the posterior contraction rate will then be $\epsilon_{n}=(n / \log n)^{(2-\alpha) /(2(1+\alpha))}$. If in addition, $\nabla f^{*}(x)=$ 0 for all $x \in \mathcal{L}^{*}$, the function then is a plateau without any rise or fall along the direction of filament, where the rate improves to $\epsilon_{n}=(n / \log n)^{(1-\alpha) /(2(1+\alpha))}$.

Theorem 5.3 below is a Bayesian analog of the convergence of filaments points on the integral curves starting from the same points; see Qiao and Polonik [30], Section 3.4, for similar results. Again a better rate is possible; see the remark following the theorem. 
Theorem 5.3. Under Assumptions (A1)-(A5) and suppose $J_{1}=J_{2}=J \asymp$ $(n / \log n)^{1 /(1+2 \alpha)}$, we have the following posterior contraction rates: for $\epsilon_{n}=$ $(n / \log n)^{(5-2 \alpha) /(2(1+2 \alpha))}$ and any $M_{n} \rightarrow \infty$,

$$
\Pi\left(\sup _{x_{0} \in \mathcal{G}}\left\|\Upsilon_{x_{0}}\left(t_{x_{0}}\right)-\Upsilon_{x_{0}}^{*}\left(t_{x_{0}}^{*}\right)\right\|>M_{n} \epsilon_{n} \mid \mathbb{D}_{n}\right) \stackrel{\mathrm{P}_{0}}{\longrightarrow} 0
$$

Remark 5.3. A better rate can be obtained using $J \asymp(n / \log n)^{1 /(2(1+\alpha))}$, giving $\epsilon_{n}=(n / \log n)^{(2-\alpha) /(2(1+\alpha))}$ in view of Remark 5.1 and Remark 5.2. In particular, the degree of smoothness of the function $f$ has been accounted for, thanks to the series approximation. For $\alpha \geq 4$, this is an improvement over the rate $n^{-1 / 6} \sqrt{\log n}$ in Theorem 3.4 of Qiao and Polonik [30] (see also their Proposition 5.1).

In the following, we shall consider the Hausdorff distance between two filaments. Given two sets $A$ and $B$ under Euclidean metric, the Hausdorff distance between $A$ and $B$ is defined as

$$
\operatorname{Haus}(A, B)=\max \{d(A \mid B), d(B \mid A)\}
$$

where $d(A \mid B):=\sup _{x \in A} \inf _{y \in B}\|x-y\|$. In what follows, we provide an upper bound for the Hausdorff distance and construct credible sets for the filaments. In fact, Theorem 5.3 gives an upper bound for the Hausdorff distance. However, for the purpose of constructing credible sets with sufficient frequentist coverage, we need to have the upper bound in terms of more primitive quantities such as the derivatives of underlying function. In view of Remark 5.3 and the fact that integral curves are only intermediate objects, we henceforth restrict to the choice $J \asymp(n / \log n)^{1 /(2(1+\alpha))}$.

Recall that $\tilde{f}=A_{(0,0)} Y+C_{(0,0)} \theta_{0}$ is the posterior mean of $f$ conditional on $\mathbb{D}_{n}$ and that $\tilde{V}, \tilde{\Upsilon}_{x_{0}}, \tilde{\mathcal{L}}$ are the corresponding eigenvector, integral curve and filament induced by $\tilde{f}$. In view of Lemma A.3 and Lemma A.4, following the proofs of Theorem 5.1, Proposition 5.2, Theorem 5.3, it is straightforward to show that $\sup _{x_{0} \in \mathcal{G}} \sup _{t}\left\|\Upsilon_{x_{0}}(t)-\tilde{\Upsilon}_{x_{0}}(t)\right\|, \sup _{x_{0} \in \mathcal{G}}\left\|t_{x_{0}}-\tilde{t}_{x_{0}}\right\|$ and $\sup _{x_{0} \in \mathcal{G}}\left\|\Upsilon_{x_{0}}\left(t_{x_{0}}\right)-\tilde{\Upsilon}_{x_{0}}\left(\tilde{t}_{x_{0}}\right)\right\|$ are all small with high posterior probability in $\mathrm{P}_{0}$-probability. Likewise, it can be shown that the quantities induced by $\tilde{f}$ converge to the corresponding true quantities induced by $f^{*}$. For instance $\tilde{\Upsilon}_{x_{0}}(t)$ converges to $\Upsilon_{x_{0}}^{*}(t)$ uniformly in $x_{0} \in \mathcal{G}$ and $t, \tilde{t}_{x_{0}}$ converges to $t_{x_{0}}^{*}$ uniformly in $x_{0} \in \mathcal{G}$ and $\tilde{\Upsilon}_{x_{0}}\left(\tilde{t}_{x_{0}}\right)$ converges to $\Upsilon_{x_{0}}^{*}\left(t_{x_{0}}^{*}\right)$ uniformly in $x_{0} \in \mathcal{G}$ in $\mathrm{P}_{0}$-probability.

The following two theorems summarize above observations. Theorem 5.4 is on the convergence rates of the Bayesian estimates of filaments to the true filaments. Theorem 5.5 is on the posterior contraction rates of filaments around filaments induced by posterior mean.

Theorem 5.4. Under Assumptions (A1), (A2) and (A5), for $J_{1}=J_{2}=J \asymp$ $(n / \log n)^{1 /(2(1+\alpha))}$, we have the following convergence rates:

$$
\sup _{x_{0} \in \mathcal{G}} \sup _{t \in\left[t_{x_{0}}^{*}-a^{*}, t_{x_{0}}^{*}+a^{*}\right]}\left\|\tilde{\Upsilon}_{x_{0}}(t)-\Upsilon_{x_{0}}^{*}(t)\right\|=O_{p}\left((n / \log n)^{\frac{2-\alpha}{2(1+\alpha)}}\right)
$$


If in addition, (A3) and (A4) hold, then

$$
\sup _{x_{0} \in \mathcal{G}}\left|\tilde{t}_{x_{0}}-t_{x_{0}}^{*}\right|=O_{p}\left((n / \log n)^{\frac{2-\alpha}{2(1+\alpha)}}\right),
$$

and

$$
\sup _{x_{0} \in \mathcal{G}}\left\|\tilde{\Upsilon}_{x_{0}}\left(\tilde{t}_{x_{0}}\right)-\Upsilon_{x_{0}}^{*}\left(t_{x_{0}}^{*}\right)\right\|=O_{p}\left((n / \log n)^{\frac{2-\alpha}{2(1+\alpha)}}\right) .
$$

Theorem 5.5. Under Assumptions (A1), (A2) and (A5), for $J_{1}=J_{2}=J \asymp$ $(n / \log n)^{1 /(2(1+\alpha))}$, we have the following posterior contraction rates: for any $M_{n} \rightarrow \infty$,

$$
\Pi\left(\sup _{x_{0} \in \mathcal{G}} \sup _{t:\left|t-t_{x_{0}}^{*}\right| \leq a^{*}}\left\|\Upsilon_{x_{0}}(t)-\tilde{\Upsilon}_{x_{0}}(t)\right\|>M_{n}(n / \log n)^{(3-2 \alpha) /(4(1+\alpha))} \mid \mathbb{D}_{n}\right) \stackrel{\mathrm{P}_{0}}{\longrightarrow} 0 .
$$

If in addition, (A3) and (A4) hold, then

$$
\Pi\left(\sup _{x_{0} \in \mathcal{G}}\left|t_{x_{0}}-\tilde{t}_{x_{0}}\right|>M_{n}(n / \log n)^{(2-\alpha) /(2(1+\alpha))} \mid \mathbb{D}_{n}\right) \stackrel{\mathrm{P}_{0}}{\longrightarrow} 0,
$$

and

$$
\Pi\left(\sup _{x_{0} \in \mathcal{G}}\left\|\Upsilon_{x_{0}}\left(t_{x_{0}}\right)-\tilde{\Upsilon}_{x_{0}}\left(\tilde{t}_{x_{0}}\right)\right\|>M_{n}(n / \log n)^{(2-\alpha) /(2(1+\alpha))} \mid \mathbb{D}_{n}\right) \stackrel{\mathrm{P}_{0}}{\longrightarrow} 0 .
$$

The following proposition says that with high posterior probability the induced filament from the posterior satisfies similar properties the true filament has, so does the induced filament by $\tilde{f}$, with $\mathrm{P}_{0}$-probability tending to one.

Proposition 5.6. Suppose that $f$ has a tensor-product B-splines prior with or$\operatorname{der} q_{1}=q_{2} \geq \alpha$ and $J \asymp(n / \log n)^{1 /(2(\alpha+1))}$, then the following assertions hold. (i) The filament $\mathcal{L}$ of $f$ drawn from the posterior distribution satisfies assumptions (A2)-(A5) with posterior probability tending to 1 under $\mathrm{P}_{0}$-probability; (ii) The induced filament $\tilde{\mathcal{L}}$ of the posterior mean $\tilde{f}$ satisfies assumptions (A2)-(A5) with $\mathrm{P}_{0}$-probability tending to 1 .

The following lemma is inspired by Genovese et al. [18] Theorem 4, where they relate the Hausdorff distance between filaments to the Euclidean distance between $V$ but under a different set of assumptions.

Lemma 5.7. Consider for two regression functions $f$ and $\hat{f}:[0,1]^{2} \mapsto \mathbb{R}$ that are sufficiently close in supremum metric and both satisfy assumptions (A1)(A5), then the Hausdorff distance between the two induced filaments satisfies, for some positive constant $c_{1}$,

$$
\operatorname{Haus}(\mathcal{L}, \hat{\mathcal{L}}) \leq \frac{c_{1}}{\eta}\left(\left\|f^{(2,0)}-\hat{f}^{(2,0)}\right\|_{\infty}+\left\|f^{(1,1)}-\hat{f}^{(1,1)}\right\|_{\infty}+\left\|f^{(0,2)}-\hat{f}^{(0,2)}\right\|_{\infty}\right) .
$$

In view of above lemma, Theorems 5.4, 5.5 and Proposition 5.6, we have the following result. 
Theorem 5.8. Under Assumptions (A1)-(A5), with the choice of $J_{1}=J_{2}=$ $J \asymp(n / \log n)^{1 /(2(\alpha+1))}$, for any $M_{n} \rightarrow \infty$,

$$
\begin{gathered}
\Pi\left(\operatorname{Haus}\left(\mathcal{L}, \mathcal{L}^{*}\right)>M_{n}(n / \log n)^{\frac{2-\alpha}{2(\alpha+1)}} \mid \mathbb{D}_{n}\right) \stackrel{\mathrm{P}_{0}}{\longrightarrow} 0, \\
\Pi\left(\operatorname{Haus}(\mathcal{L}, \tilde{\mathcal{L}})>M_{n}(n / \log n)^{\frac{2-\alpha}{2(\alpha+1)}} \mid \mathbb{D}_{n}\right) \stackrel{\mathrm{P}_{0}}{\longrightarrow} 0,
\end{gathered}
$$

and

$$
\operatorname{Haus}\left(\tilde{\mathcal{L}}, \mathcal{L}^{*}\right)=O_{p}\left((n / \log n)^{\frac{2-\alpha}{2(\alpha+1)}}\right) .
$$

Remark 5.4. The third assertion of Theorem 5.8 for the convergence rate of the filament induced by the posterior mean is an improvement over the rate $(\log n / n)^{1 / 5}$ in Theorem 5 of Genovese et al. [18] when $\alpha>4$.

For the following result, we restrict multi-index $r$ to the collection $\mathcal{R}:=$ $\{(2,0),(1,1),(0,2)\}$. Let $\tilde{f}^{(r)}:=A_{r} Y+C_{r} \theta_{0}$ be the posterior mean of $f^{(r)}$ and $\mathcal{L}$ be the induced filament. For some $0<\gamma<1 / 2$, let $R_{n, r, \gamma}$ denote the $1-\gamma$ quantile of the posterior distribution of $\left\|f^{(r)}-\tilde{f}^{(r)}\right\|_{\infty}$. Let $C_{f, r, \gamma}^{\rho}:=\{f$ : $\left.\left\|f^{(r)}-\tilde{f}^{(r)}\right\|_{\infty} \leq \rho R_{n, r, \gamma}\right\}$ for some large $\rho>1$. The following theorem provides two valid credible sets with sufficiently high frequentist coverage as the sample size increases. The choices of $c_{1}, \eta$ and $\rho$ will be discussed in the next section.

Theorem 5.9. Assume (A1)-(A5), for $J_{1}=J_{2}=J \asymp(n / \log n)^{1 /(2(\alpha+1))}$ and some sufficiently large constant $\rho>1$, for the following two sets,

$$
\begin{aligned}
C_{\mathcal{L}} & =\left\{\mathcal{L}(f): f \in \cap_{r \in \mathcal{R}} C_{f, r, \gamma}^{\rho}\right\}, \\
\bar{C}_{\mathcal{L}} & =\left\{\mathcal{L}: \operatorname{Haus}(\mathcal{L}, \tilde{\mathcal{L}}) \leq \frac{c_{1}}{\eta} \rho \max _{r \in \mathcal{R}} R_{n, r, \gamma}\right\},
\end{aligned}
$$

the credibility of $C_{\mathcal{L}}$ and its coverage probability for $\mathcal{L}^{*}$ tend to 1 and $C_{\mathcal{L}} \subset \bar{C}_{\mathcal{L}}$ with high posterior probability with $\mathrm{P}_{0}$-probability tending to 1 .

\section{Simulation}

Many algorithms have been proposed to find filaments. We here use an algorithm that shares a similar spirit of the Subspace Constrained Mean Shift (SCMS) algorithm proposed in Ozertem and Erdogmus [29]. SCMS algorithm was also used in Genovese et al. [18], Chen, Genovese and Wasserman [6] and Chen et al. [7]. The key of the algorithm is to project the gradient onto the direction given by $V$. Even though in the literature the algorithm is primarily used with kernel density estimator, our study suggests that nothing hinders the efficacy of the algorithm when applied with a series based estimation in either regression or density estimation setting. In the following, we give a description of the algorithm.

Algorithm: (Subspace Constrained Gradient Ascent Algorithm)

Set $\epsilon>0, \tau>0, \bar{a}>0$ and select a collection of points $\left\{x_{1}, \ldots, x_{n}\right\}$, compute $f\left(x_{i}\right)$ and keep only those points for which $f\left(x_{i}\right)>\tau$. For each $x_{i}$, let $x_{i}^{(1)}=x_{i}$. Now iterate through the following steps starting from $t=1$ : 
(1) evaluate $\nabla f\left(x_{i}^{(t)}\right)$;

(2) evaluate the Hessian $H f\left(x_{i}^{(t)}\right)$ and perform spectral decomposition to get $V\left(x_{i}^{(t)}\right)$ the normalized eigenvector of $H f\left(x_{i}^{(t)}\right)$ with the smallest eigenvalue;

(3) update $x_{i}^{(t+1)}=\bar{a} V\left(x_{i}^{(t)}\right) V^{T}\left(x_{i}^{(t)}\right) \nabla f\left(x_{i}^{(t)}\right)+x_{i}^{(t)}$;

(4) stop if $\left\|x_{i}^{(t+1)}-x_{i}^{(t)}\right\|<\epsilon$ or $\left|V^{T}\left(x_{i}^{(t)}\right) \nabla f\left(x_{i}^{(t)}\right)\right|<\epsilon$.

If stop at $t=t^{*}$, keep the point $x_{i}^{\left(t^{*}\right)}$ that satisfies $\lambda\left(x_{i}^{\left(t^{*}\right)}\right)<0$.

In the simulation, we consider the following function

$$
f\left(x_{1}, x_{2}\right)=1+\left(\phi\left(\sqrt{x_{1}^{2}+x_{2}^{2}}\right)\right)^{1+\cos ^{2}\left(\tan ^{-1}\left(x_{2} / x_{1}\right)\right)},
$$

where $\phi(\cdot)$ is the normal density function with mean 0.5 and standard deviation 0.3 . We generate i.i.d. data $X_{i}$ uniformly on $[0,1] \times[0,1]$ and i.i.d. $\varepsilon_{i}$ from normal with mean 0 and standard deviation 0.1 and then set $Y_{i}=f\left(X_{i, 1}, X_{i, 2}\right)+\epsilon_{i}$. The sample size is 2000. The Figure 1 shows function $f$ and its filament. The smoothing parameter $\left(J_{1}, J_{2}\right)$ plays an important role as in any nonparametric estimation problem.

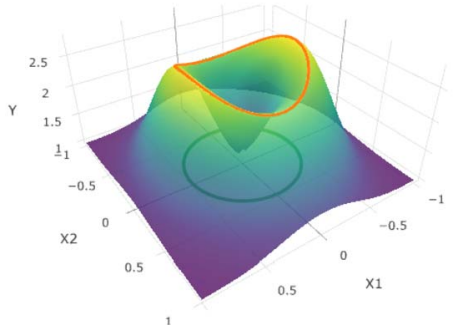

FIG 1. The function $f$ and its filament.

We use fifth-order B-splines functions, that is $q_{1}=q_{2}=5$. One can choose the pair $\left(J_{1}, J_{2}\right)$ by their posterior mode (in a logarithmic scale) by maximizing the following

$$
\log \Pi\left(J_{1}, J_{2} \mid \mathbb{D}_{n}\right)=-2 n \log \hat{\sigma}-\log \left(\operatorname{det}\left(B \Lambda_{0} B^{T}\right)+I_{n}\right)+\text { const. }
$$

We use $\tau=2, \bar{a}=0.02$ and $\epsilon=10^{-6}$. Some pilot simulation suggests that $\left(J_{1}, J_{2}\right)=(9,9)$ is picked by its posterior mode throughout. Different choices have been experimented as well. In general, "oversmoothing" may distort the filaments, whereas "undersmoothing" seems to produce similar results as using $J_{1}=J_{2}=9$, as can be seen in Figure 2 . We also provide uncertainty quantification in Figure 3 with $\gamma=0.1, \rho=1.2$. Each graph shows 100 posterior filaments drawn from $C_{\mathcal{L}}$. To evaluate $R_{n, r, \gamma}$ for $r \in \mathcal{R}=\{(2,0),(1,1),(0,2)\}$, we first draw 200 posterior samples of $\theta$, compute their posterior mean $\tilde{\theta}$. Next we compute $\sup _{x}\left|b_{J_{1}, J_{2}}^{(r)}(x)^{T}(\theta-\tilde{\theta})\right|$ by searching on a crude grid and pick the maximum 
point on the grid and then starting from this maximum point apply gradient ascent or descent method to check if nearby points can achieve greater (absolute) value. We keep the largest value as the supremum. The $(1-\gamma)$-empirical quantile over all these suprema will then be our $R_{n, r, \gamma}$. The filaments from the posterior samples that fall in the set $C_{\mathcal{L}}$ can then be generated.
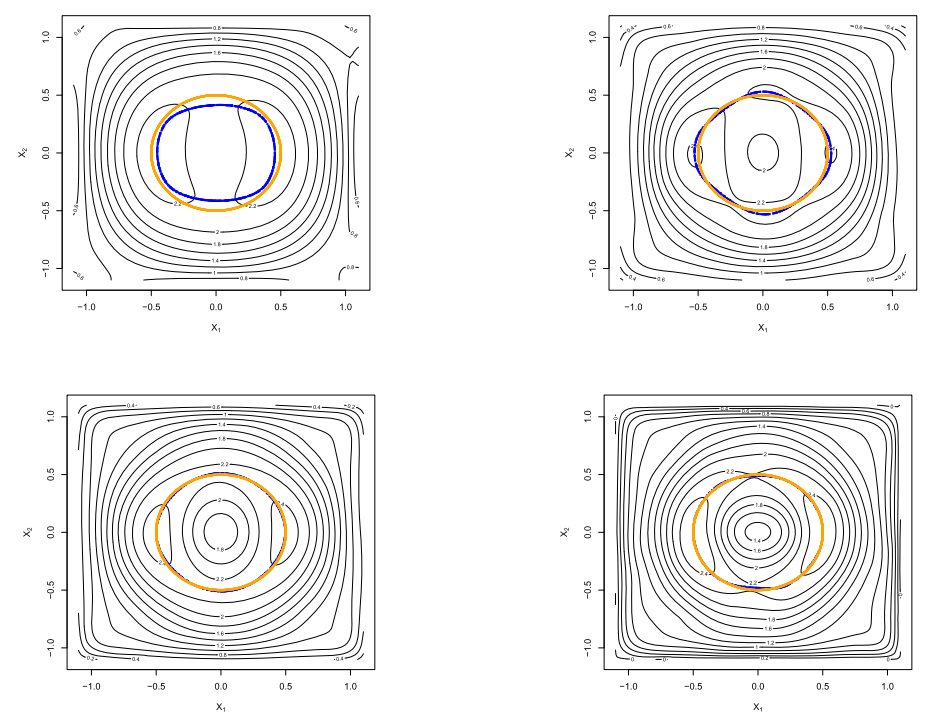

FIG 2. Effects of the smoothing parameter $J_{1}$ and $J_{2}$. The orange circle is the truth, blue curve (consists of dots) is estimated filament induced by posterior mean. Top left: $J_{1}=J_{2}=7$; top right: $J_{1}=J_{2}=8$; bottom left: $J_{1}=J_{2}=9$; bottom right: $J_{1}=J_{2}=15$.
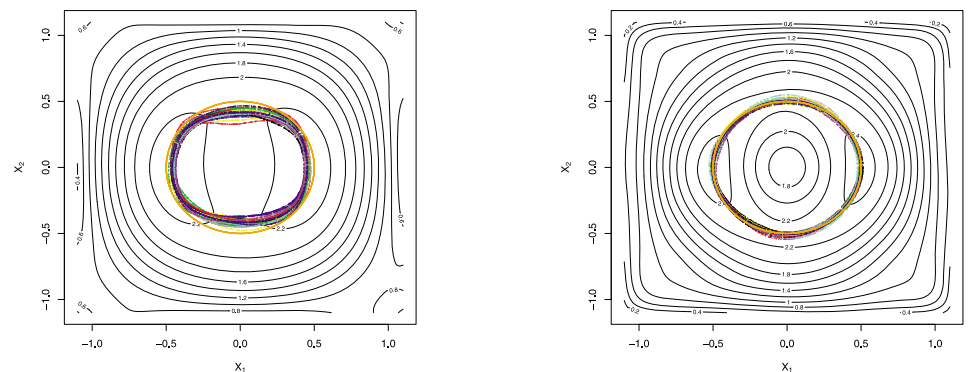

FIG 3. Uncertainty Quantification. Left: $J_{1}=J_{2}=7$. Right: $J_{1}=J_{2}=9$.

To assess the performance over 100 iterations, we compute the Hausdorff distance between $\mathcal{L}^{*}$ and $\tilde{\mathcal{L}}$. The value of $\rho$ should not be too large but gives a reasonable high percentage of $f$ from posterior that fall in $C_{f, r, \gamma}^{\rho}$. In practice, reasonable values of $\rho$ can be calibrated by some pilot simulation using the posterior samples. For instance, $\rho=1.2$ is a reasonable choice in this simulation, 
giving $92.33 \%$ credibility averaging over all iterations. To evaluate the coverage performance, we compute the Hausdorff distance between $\mathcal{L}^{*}$ and $\tilde{\mathcal{L}}$. From the definition of $\bar{C}_{\mathcal{L}}$, we set $c_{1} / \eta$ to different values. Simulation shows that $\mathcal{L}^{*}$ belongs to $\bar{C}_{\mathcal{L}}$ for $91 \%, 94 \%, 98 \%$ time when $c_{1} / \eta$ takes value $7.3 \times 10^{-4}, 7.5 \times 10^{-4}$ and $8 \times 10^{-4}$ respectively. In practice, $c_{1}$ can be computed using $\sup _{x}\|\nabla \tilde{f}(x)\|$ and $\eta$ as the smallest value of $-\lambda$ along the filament induced by the posterior mean. With this method, we obtain $100 \%$ coverage - high coverage as the theory predicts.

\section{Application}

For application, we use an earthquake dataset for California and its vicinity from January 1st of 2013 to December 31th of 2017 with magnitude 3.0 and above on the Richter scale ${ }^{1}$. The dataset consists of 3772 observations, among which 3383 observations have magnitude between 3 and 4; 355 observations between 4 and 5; 34 observations above 5 . The average magnitude is 3.439 . The left panel in Figure 4 shows the data scatter plot. The sizes of circles are proportional to the magnitudes of the earthquakes.

In the algorithm, we use $\bar{a}=5 \times 10^{-6}$ and $\tau=3$ and $\epsilon=10^{-6}$. We use $q_{1}=q_{2}=4$ and $J_{1}=J_{2}=32$. We draw 200 posterior samples to compute the posterior mean. The filaments induced by the posterior mean is plotted as the blue curve in Figure 4. The same filaments are overlayed on the magnitude surface as given in Figure 5. To obtain uncertainty quantification using filaments from posterior samples, we use $\gamma=0.1$ (i.e., $90 \%$ credibility), $\rho=1.2$. The results showed that there are $91 \%$ of posterior realizations fall into $C_{\mathcal{L}}$, only slightly higher than the nominal credibility level. We randomly pick 100 of them to describe the uncertainty quantification as given in the right panel of Figure 4 .
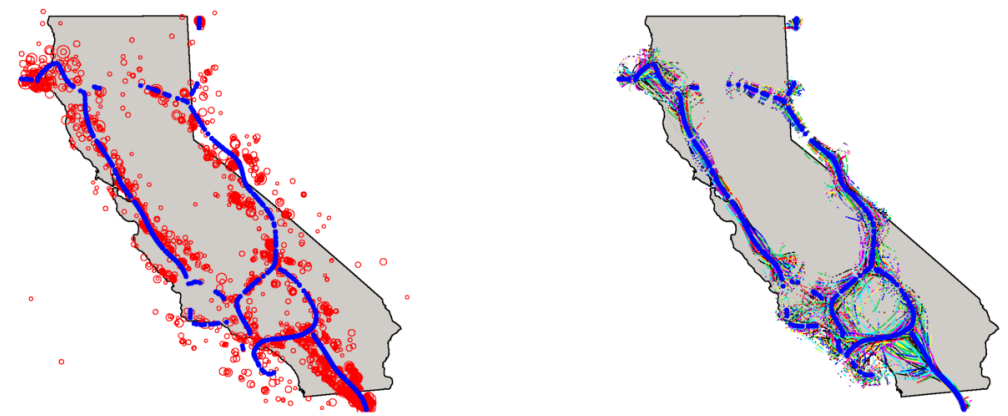

FIG 4. Left: Earthquake data points (in red). Right: 100 filaments in the posterior constructed with high frequentist coverage. The thick blue curves are the filaments induced by the posterior mean.

\footnotetext{
${ }^{1}$ The data is publicly available from https://earthquake.usgs.gov/earthquakes/.
} 


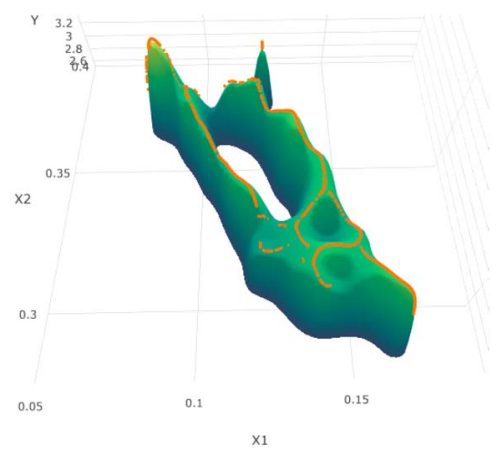

FIG 5. The magnitude surface and filaments induced by the posterior mean (traces on the graph).

The filaments hence obtained provide useful characterization of the features of earthquake magnitude. Geographically, these filaments pass through the most populous coastal urban and suburban areas in California, for instance, Eureka city, San Francisco and Los Angeles. Since this application has utilized very small portion of earthquake data, we believe that a large scale study for different periods of historical times will be useful for the study of the dynamics of the earthquakes. Uncertainty quantification provides the statistical understanding of what might be considered as reasonable shifts of these filaments through spatial and temporal domain and are also helpful for discovering newly emerging crustal activities.

\section{Appendix A: Technical proofs}

In this section, we will provide lemmas and formal proofs of the results stated in the main text. To focus on discussion, we will draw on several useful results directly given in the following remark.

Remark A.1. Under the assumptions (A1) to (A5), the following assertions hold.

(1) Integral curves are dense and non-overlapping as starting points vary; the set $\mathcal{G}$ is compact.

(2) $x_{0} \mapsto t_{x_{0}}^{*}$ is continuous.

(3) $\left\|\Upsilon_{x_{0}}^{*}(t)-\Upsilon_{x_{0}^{\prime}}^{*}(t)\right\| \leq C \exp (t)\left\|x_{0}-x_{0}^{\prime}\right\|$ for some constant $C>0$.

(4) $G=\left(G_{1}, G_{2}\right)^{T}, \nabla G$ are Lipschitz continuous and each element of Hessian $H G_{1}, H G_{2}$ is bounded on some open set $Q_{\delta} \subset \mathbb{R}^{3}$ such that $\left\{d^{2} f^{*}(x)\right.$ : $\left.x \in[0,1]^{2}\right\} \subset Q_{\delta}$. Thus $V^{*}$ is Lipschitz continuous.

There results can be proved using arguments similar to Qiao and Polonik [30]. The original argument and proofs appear in that paper in various places. To save space, we do not provide the details here, but point to these following 
specific places in their paper. Result (1) is given in the discussion in page 10, result (2) and (3) are discussed in pages 22 and 48, and result (4) is discussed in pages $53-55$.

We shall also use the following result frequently in our proofs.

Remark A.2. If the Condition (3.1) holds, then we have

$$
C_{1} n\left(\prod_{k=1}^{2} J_{k}^{-1}\right) I_{J_{1} J_{2}} \leq B^{T} B \leq C_{2} n\left(\prod_{k=1}^{2} J_{k}^{-1}\right) I_{J_{1} J_{2}}
$$

for some constants $C_{1}, C_{2}>0$. If in addition, for some constants $0<c_{1} \leq c_{2}<$ $\infty$ such that

$$
c_{1} I_{J_{1} J_{2}} \leq \Lambda \leq c_{2} I_{J_{1} J_{2}}
$$

we have

$$
\left(C_{1} n \prod_{k=1}^{2} J_{k}^{-1}+c_{2}^{-1}\right) I_{J_{1} J_{2}} \leq B^{T} B+\Lambda^{-1} \leq\left(C_{2} n \prod_{k=1}^{2} J_{k}^{-1}+c_{1}^{-1}\right) I_{J_{1} J_{2}} .
$$

Proof. See Yoo and Ghosal [38] Lemma A.9. and the discussion in p. 1075.

\section{A.1. Some lemmas}

Lemma A.1. For $f \in \mathcal{H}^{\alpha}\left([0,1]^{2}\right)$ with $\alpha \leq \min \left(q_{1}, q_{2}\right)$. Then for $J_{1}, J_{2}$ sufficiently large, there exists a function $f^{\infty}:=b_{J_{1}, J_{2}}^{T} \theta_{\infty}$ for some $\theta_{\infty}$ such that

$$
\left\|f^{\infty}-f\right\|_{\infty} \leq C \sum_{k=1}^{2} J_{k}^{-\alpha}, \quad\left\|D^{r} f^{\infty}-D^{r} f\right\|_{\infty} \leq C \sum_{k=1}^{2} J_{k}^{r_{k}-\alpha},
$$

for some positive constant $C$ depending on $\alpha$ and $q$ and for every integer vector $r$ satisfying $|r|<\alpha$.

Proof. For proof, see Schumaker [32].

Lemma A.2 (Posterior contraction around truth). Under the above assumptions and $J_{1}=J_{2}=J$ and $J^{2} \leq n$, with $J$ chosen such that $\delta_{n, k, J}$ vanishes to zero, then for any $M_{n} \rightarrow \infty$,

$$
\begin{aligned}
& \Pi\left(\sup _{x \in[0,1]^{2}}\left|D^{r} f(x)-D^{r} f^{*}(x)\right|>M_{n} \delta_{n,|r|, J} \mid \mathbb{D}_{n}\right) \stackrel{\mathrm{P}_{0}}{\longrightarrow} 0, \\
& \Pi\left(\sup _{x \in[0,1]^{2}}\left\|\nabla f(x)-\nabla f^{*}(x)\right\|>M_{n} \delta_{n, 1, J} \mid \mathbb{D}_{n}\right) \stackrel{\mathrm{P}_{0}}{\longrightarrow} 0, \\
& \Pi\left(\sup _{x \in[0,1]^{2}}\left\|d^{2} f(x)-d^{2} f^{*}(x)\right\|>M_{n} \delta_{n, 2, J} \mid \mathbb{D}_{n}\right) \stackrel{\mathrm{P}_{0}}{\longrightarrow} 0, \\
& \Pi\left(\sup _{x \in[0,1]^{2}}\left\|H f(x)-H f^{*}(x)\right\|_{F}>M_{n} \delta_{n, 2, J} \mid \mathbb{D}_{n}\right) \stackrel{\mathrm{P}_{0}}{\longrightarrow} 0,
\end{aligned}
$$




$$
\begin{aligned}
& \Pi\left(\sup _{x \in[0,1]^{2}}\left\|\nabla d^{2} f(x)-\nabla d^{2} f^{*}(x)\right\|_{F}>M_{n} \delta_{n, 3, J} \mid \mathbb{D}_{n}\right) \stackrel{\mathrm{P}_{0}}{\longrightarrow} 0, \\
& \Pi\left(\sup _{x \in[0,1]^{2}}\left\|V(x)-V^{*}(x)\right\|_{F}>M_{n} \delta_{n, 2, J} \mid \mathbb{D}_{n}\right) \stackrel{\mathrm{P}_{0}}{\longrightarrow} 0, \\
& \Pi\left(\sup _{x \in[0,1]^{2}}\left\|\nabla V(x)-\nabla V^{*}(x)\right\|_{F}>M_{n} \delta_{n, 3, J} \mid \mathbb{D}_{n}\right) \stackrel{\mathrm{P}_{0}}{\longrightarrow} 0 .
\end{aligned}
$$

where $\delta_{n, k, J}=J^{k}\left((\log n / n) J^{2}+J^{-2 \alpha}\right)^{1 / 2}$.

Proof. These results can be directly adapted from Yoo and Ghosal [38] by noting the highest degree of derivatives in each expression. We shall show the rates for $\sup _{x}\left\|H f(x)-H f^{*}(x)\right\|_{F}, \sup _{x}\left\|\nabla d^{2} f(x)-\nabla d^{2} f^{*}(x)\right\|_{F}$ and $\sup _{x} \| \nabla V(x)-$ $\nabla V^{*}(x) \|_{F}$. Notice that $\left\|H f(x)-H f^{*}(x)\right\|_{F}$ is equal to

$$
\begin{aligned}
& \left(\left(f^{(2,0)}(x)-f^{*(2,0)}(x)\right)^{2}+2\left(f^{(1,1)}(x)-f^{*(1,1)}(x)\right)^{2}\right. \\
& \left.\quad+\left(f^{(0,2)}(x)-f^{*(0,2)}(x)\right)^{2}\right)^{1 / 2}
\end{aligned}
$$

which can be bounded by

$4^{-1 / 2}\left(\left|f^{(2,0)}(x)-f^{*(2,0)}(x)\right|+2\left|f^{(1,1)}(x)-f^{*(1,1)}(x)\right|+\left|f^{(0,2)}(x)-f^{*(0,2)}(x)\right|\right)$.

The rate for $\sup _{x}\left\|H f(x)-H f^{*}(x)\right\|_{F}$ then follows easily.

Also, the contraction rates for $\left\|\nabla d^{2} f(x)-\nabla d^{2} f^{*}(x)\right\|_{F}$ follows from the inequality $\left\|\nabla d^{2} f(x)-\nabla d^{2} f^{*}(x)\right\|_{F} \leq 6^{-1 / 2} \sum_{r:|r|=3}\left|D^{r} f(x)-D^{r} f^{*}(x)\right|$.

Lastly, since $\nabla V(x)-\nabla V^{*}(x)=\nabla G\left(d^{2} f(x)\right) \nabla d^{2} f(x) \quad$ $\nabla G\left(d^{2} f^{*}(x)\right) \nabla d^{2} f^{*}(x)$, which is 2 by 2 matrix. Straightforward calculation gives its $(1,1)$ element

$$
\begin{aligned}
& \left(G_{1}^{(1,0,0)}\left(d^{2} f(x)\right) f^{(3,0)}(x)-G_{1}^{(1,0,0)}\left(d^{2} f^{*}(x)\right) f^{*(3,0)}(x)\right), \\
+ & \left(G_{1}^{(0,1,0)}\left(d^{2} f(x)\right) f^{(2,1)}(x)-G_{1}^{(0,1,0)}\left(d^{2} f^{*}(x)\right) f^{*(2,1)}(x)\right), \\
+ & \left(G_{1}^{(0,0,1)}\left(d^{2} f(x)\right) f^{(1,2)}(x)-G_{1}^{(0,0,1)}\left(d^{2} f^{*}(x)\right) f^{*(1,2)}(x)\right) .
\end{aligned}
$$

The absolute value of the first summand is bounded by the sum of

$$
\left|G_{1}^{(1,0,0)}\left(d^{2} f(x)\right) f^{(3,0)}(x)-G_{1}^{(1,0,0)}\left(d^{2} f(x)\right) f^{*(3,0)}(x)\right|,
$$

and

$$
\left|G_{1}^{(1,0,0)}\left(d^{2} f(x)\right) f^{*(3,0)}(x)-G_{1}^{(1,0,0)}\left(d^{2} f^{*}(x)\right) f^{*(3,0)}(x)\right| .
$$

Noting that $d^{2} f(x)$ contracts to $d^{2} f^{*}(x)$ uniformly in $x$, hence $\left\{d^{2} f(x): x \in\right.$ $\left.[0,1]^{2}\right\} \subset Q_{\delta}$ with posterior probability tending to 1 , the first term is bounded by a constant multiple of $\left|f^{(3,0)}(x)-f^{*(3,0)}(x)\right|$, in view of the result (4) of 
Remark A.1. By the same remark and assumption $\left\|f^{*}\right\|_{\alpha, \infty}<\infty$, the second term is bounded by $\left\|d^{2} f(x)-d^{2} f^{*}(x)\right\|\left|f^{*(3,0)}(x)\right|$. Using similar arguments for the second and third summand, one can see that the absolute value of the $(1,1)$ th element of $\nabla V(x)-\nabla V^{*}(x)$ is bounded by $\left|f^{(3,0)}(x)-f^{*(3,0)}(x)\right|+\mid f^{(2,1)}(x)-$ $f^{*(2,1)}(x)|+| f^{(1,2)}(x)-f^{*(1,2)}(x) \mid$. Dealing the remaining elements of $\nabla V(x)-$ $\nabla V^{*}(x)$ similarly, we have that $\left\|\nabla V(x)-\nabla V^{*}(x)\right\|_{F} \lesssim \sum_{r:|r|=3} \mid D^{r} f(x)-$ $D^{r} f^{*}(x) \mid$.

In above lemma, the optimal rates are obtained when $J \asymp(n / \log n)^{1 / 2(\alpha+1)}$, which then yields

$$
\delta_{n, k, J}=\epsilon_{n} \asymp(\log n / n)^{(\alpha-k) /(2 \alpha+2)} .
$$

In addition, we have the following two lemmas whose proofs closely follow that of Lemma A.2 and thus are omitted. Denote the posterior mean of $f$ by $\tilde{f}:=A_{(0,0)} Y+C_{(0,0)} \theta_{0}$ and similarly define the quantities it induces, for instance, $\tilde{V}, \tilde{\Upsilon}_{x_{0}}$ and $\tilde{\mathcal{L}}$. We then have the following lemma.

Lemma A.3 (Posterior contraction around the posterior mean). Under the above assumptions and $J_{1}=J_{2}=J$ and $J^{2} \leq n$, with $J$ chosen such that $\eta_{n, k, J}$ vanishes to zero, then for any $M_{n} \rightarrow \infty$,

$$
\begin{aligned}
& \Pi\left(\sup _{x \in[0,1]^{2}}\left|D^{r} f(x)-D^{r} \tilde{f}(x)\right|>M_{n} \eta_{n,|r|, J} \mid \mathbb{D}_{n}\right) \stackrel{\mathrm{P}_{0}}{\longrightarrow} 0, \\
& \Pi\left(\sup _{x \in[0,1]^{2}}\|\nabla f(x)-\nabla \tilde{f}(x)\|>M_{n} \eta_{n, 1, J} \mid \mathbb{D}_{n}\right) \stackrel{\mathrm{P}_{0}}{\longrightarrow} 0, \\
& \Pi\left(\sup _{x \in[0,1]^{2}}\left\|d^{2} f(x)-d^{2} \tilde{f}(x)\right\|>M_{n} \eta_{n, 2, J} \mid \mathbb{D}_{n}\right) \stackrel{\mathrm{P}_{0}}{\longrightarrow} 0, \\
& \Pi\left(\sup _{x \in[0,1]^{2}}\|H f(x)-H \tilde{f}(x)\|_{F}>M_{n} \eta_{n, 2, J} \mid \mathbb{D}_{n}\right) \stackrel{\mathrm{P}_{0}}{\longrightarrow} 0, \\
& \Pi\left(\sup _{x \in[0,1]^{2}}\left\|\nabla d^{2} f(x)-\nabla d^{2} \tilde{f}(x)\right\|_{F}>M_{n} \eta_{n, 3, J} \mid \mathbb{D}_{n}\right) \stackrel{\mathrm{P}_{0}}{\longrightarrow} 0, \\
& \Pi\left(\sup _{x \in[0,1]^{2}}\|V(x)-\tilde{V}(x)\|_{F}>M_{n} \eta_{n, 2, J} \mid \mathbb{D}_{n}\right) \stackrel{\mathrm{P}_{0}}{\longrightarrow} 0, \\
& \Pi\left(\sup _{x \in[0,1]^{2}}\|\nabla V(x)-\nabla \tilde{V}(x)\|_{F}>M_{n} \eta_{n, 3, J} \mid \mathbb{D}_{n}\right) \stackrel{\mathrm{P}_{0}}{\longrightarrow} 0,
\end{aligned}
$$

where $\eta_{n, k, J}=J^{k+1}(\log n / n)^{1 / 2}$.

One can also readily obtain the following convergence rates for the Bayesian estimators induced by $\tilde{f}$.

Lemma A.4 (Convergence of posterior mean). Under the above assumptions and $J_{1}=J_{2}=J$ and $J^{2} \leq n$, with $J$ chosen such that $\delta_{n, k, J}$ vanishes to zero, then we have

$$
\sup _{x \in[0,1]^{2}}\left|D^{r} \tilde{f}(x)-D^{r} f^{*}(x)\right|=O_{p}\left(\delta_{n,|r|, J}\right),
$$




$$
\begin{aligned}
& \sup _{x \in[0,1]^{2}}\left\|\nabla \tilde{f}(x)-\nabla f^{*}(x)\right\|=O_{p}\left(\delta_{n, 1, J}\right), \\
& \sup _{x \in[0,1]^{2}}\left\|d^{2} \tilde{f}(x)-d^{2} f^{*}(x)\right\|=O_{p}\left(\delta_{n, 2, J}\right), \\
& \sup _{x \in[0,1]^{2}}\left\|H \tilde{f}(x)-H f^{*}(x)\right\|_{F}=O_{p}\left(\delta_{n, 2, J}\right), \\
& \sup _{x \in[0,1]^{2}}\left\|\nabla d^{2} \tilde{f}(x)-\nabla d^{2} f^{*}(x)\right\|_{F}=O_{p}\left(\delta_{n, 3, J}\right), \\
& \sup _{x \in[0,1]^{2}}\left\|\tilde{V}(x)-V^{*}(x)\right\|_{F}=O_{p}\left(\delta_{n, 2, J}\right), \\
& \sup _{x \in[0,1]^{2}}\left\|\nabla \tilde{V}(x)-\nabla V^{*}(x)\right\|_{F}=O_{p}\left(\delta_{n, 3, J}\right),
\end{aligned}
$$

where $\delta_{n, k, J}=J^{k}\left((\log n / n) J^{2}+J^{-2 \alpha}\right)^{1 / 2}$.

We will also need the following lemma in the proof of Theorem 5.1.

Lemma A.5. Let $\tilde{G}\left(\Upsilon_{x_{0}}^{*}(s)\right)=\frac{\partial G_{1}}{\partial x_{1}}\left(d^{2} f^{*}\left(\Upsilon_{x_{0}}^{*}(s)\right)\right)$. For any $x_{0}, \tilde{x}_{0} \in \mathcal{G}$, let $T_{x_{0}}^{*}=t_{x_{0}}^{*}+a^{*}, T_{\tilde{x}_{0}}^{*}=t_{\tilde{x}_{0}}^{*}+a^{*}, t \in\left[0, T_{x_{0}}^{*}\right]$ and $\tilde{t} \in\left[0, T_{\tilde{x}_{0}}^{*}\right]$. Define $a\left(x_{0}, t\right):=$ $\int_{0}^{t} \tilde{G}\left(\Upsilon_{x_{0}}^{*}(s)\right) b_{J_{1}, J_{2}}^{(r)}\left(\Upsilon_{x_{0}}^{*}(s)\right) d s$ for $r=(2,0),(1,1)$ or $(0,2)$, where the integral is taken elementwise. Under Assumption (A1), (A2) and (A5) and $J_{1}=J_{2}=J$, we have

$$
\begin{aligned}
& \left\|a\left(x_{0}, t\right)\right\|_{1} \lesssim J^{2}, \quad\left\|a\left(x_{0}, t\right)\right\|^{2} \lesssim J^{3} \\
& \left\|a\left(x_{0}, t\right)-a\left(\tilde{x}_{0}, \tilde{t}\right)\right\|^{2} \lesssim J^{3}|t-\tilde{t}|+J^{5}\left\|x_{0}-\tilde{x}_{0}\right\|^{2} .
\end{aligned}
$$

\section{A.2. Proofs of the main results}

Since $\Upsilon_{x_{0}}(-t)=\int_{0}^{t}\left(-V\left(\Upsilon_{x_{0}}(-s)\right)\right) d s$, with negative time it can be interpreted as a curve tracing in the reverse direction, i.e, $-V$. Since the direction of $V$ does not play a role in the theoretical proof, without loss of generality, $t$ is restricted on the $\left[0, T_{x_{0}}^{*}\right]$ where $T_{x_{0}}^{*}=t_{x_{0}}^{*}+a^{*}$ and we shall assume the hitting times are nonnegative.

Proof of Theorem 5.1. We first sketch a proof for the following result as first proved in Koltchinskii et al. [24]:

$$
\sup _{x_{0} \in \mathcal{G}} \sup _{t \in\left[0, T_{x_{0}}^{*}\right]}\left\|\Upsilon_{x_{0}}(t)-\Upsilon_{x_{0}}^{*}(t)\right\| \lesssim \sup _{x_{0} \in \mathcal{G}} \sup _{t \in\left[0, T_{x_{0}}^{*}\right]}\left\|\int_{0}^{t}\left(V-V^{*}\right)\left(\Upsilon_{x_{0}}^{*}(s)\right) d s\right\| .
$$

To see this, let

$$
\begin{aligned}
& y_{x_{0}}(t):=\Upsilon_{x_{0}}(t)-\Upsilon_{x_{0}}^{*}(t)=\int_{0}^{t}\left(V\left(\Upsilon_{x_{0}}(s)\right)-V^{*}\left(\Upsilon_{x_{0}}^{*}(s)\right) d s\right. \\
& z_{x_{0}}(t):=\int_{0}^{t}\left(V-V^{*}\right) \Upsilon_{x_{0}}^{*}(s) d s+\int_{0}^{t} \nabla V^{*}\left(\Upsilon_{x_{0}}^{*}(s)\right) z_{x_{0}}(s) d s
\end{aligned}
$$


Note that $\delta_{x_{0}}(t):=y_{x_{0}}(t)-z_{x_{0}}(t)=\int_{0}^{t} \nabla V^{*}\left(\Upsilon_{x_{0}}^{*}(s)\right) \delta(s) d s+R_{x_{0}}(t)$ for some reminder term $R_{x_{0}}(t)$. So $\left\|\delta_{x_{0}}(t)\right\| \leq\left\|R_{x_{0}}(t)\right\|+\int_{0}^{t}\left\|\nabla V^{*}\left(\Upsilon_{x_{0}}^{*}(s)\right)\right\|_{F}\left\|\delta_{x_{0}}(s)\right\| d s$, by the Gronwall-Bellman inequality (Kim [22]),

$$
\left\|\delta_{x_{0}}(t)\right\| \leq \sup _{t \in\left[0, T_{x_{0}}^{*}\right]}\left(\left\|R_{x_{0}}(t)\right\| \exp \left(\int_{0}^{t}\left\|\nabla V^{*}\left(\Upsilon_{x_{0}}^{*}(s)\right)\right\|_{F} d s\right)\right) .
$$

Hence $\left.\sup _{t \in\left[0, T_{x_{0}}^{*}\right]}\left\|\delta_{x_{0}}(t)\right\|\right) \lesssim \sup _{t \in\left[0, T_{x_{0}}^{*}\right]}\left\|R_{x_{0}}(t)\right\|$. It holds also that with high posterior probability $\sup _{t \in\left[0, T_{x_{0}}^{*}\right]}\left\|R_{x_{0}}(t)\right\| \ll \int_{0}^{T_{x_{0}}^{*}}\left\|y_{x_{0}}(s)\right\| d s$ in $\mathrm{P}_{0}$-probability tending to 1 following the argument in page 1586 of Koltchinskii et al. [24]. Therefore, $\sup _{t \in\left[0, T_{x_{0}}^{*}\right]}\left\|\delta_{x_{0}}(t)\right\| \ll \int_{0}^{T_{x_{0}}^{*}}\left\|y_{x_{0}}(s)\right\| d s$. Since $y_{x_{0}}(t)=\delta_{x_{0}}(t)+z_{x_{0}}(t)$, it follows that $\sup _{t \in\left[0, T_{x_{0}}^{*}\right]}\left\|\delta_{x_{0}}(t)\right\| \ll \int_{0}^{T_{x_{0}}^{*}}\left\|z_{x_{0}}(s)\right\| d s \lesssim \sup _{t \in\left[0, T_{x_{0}}^{*}\right]}\left\|z_{x_{0}}(t)\right\|$. Then $\sup _{t \in\left[0, T_{x_{0}}^{*}\right]}\left\|y_{x_{0}}(t)\right\|=\sup _{t \in\left[0, T_{x_{0}}^{*}\right]}\left\|\delta_{x_{0}}(t)+z_{x_{0}}(t)\right\| \lesssim \sup _{t \in\left[0, T_{x_{0}}^{*}\right]}\left\|z_{x_{0}}(t)\right\|$. But

$$
\left\|z_{x_{0}}(t)\right\| \leq\left\|\int_{0}^{t}\left(V-V^{*}\right) \Upsilon_{x_{0}}^{*}(s) d s\right\|+\int_{0}^{t}\left\|\nabla V^{*}\left(\Upsilon_{x_{0}}^{*}(s)\right)\right\|_{F}\left\|z_{x_{0}}(s)\right\| d s .
$$

One more application of the Gronwall-Bellman inequality and then taking the supremum on the left hand side yields

$$
\begin{aligned}
\sup _{t}\left\|z_{x_{0}}(t)\right\| & \leq \sup _{t}\left(\left\|\int_{0}^{t}\left(V-V^{*}\right) \Upsilon_{x_{0}}^{*}(s) d s\right\| \exp \left(\int_{0}^{t}\left\|\nabla V^{*}\left(\Upsilon_{x_{0}}^{*}(s)\right)\right\|_{F} d s\right)\right) \\
& \lesssim \sup _{t}\left\|\int_{0}^{t}\left(V-V^{*}\right) \Upsilon_{x_{0}}^{*}(s) d s\right\| .
\end{aligned}
$$

Taking another supremum over all $x_{0}$ gives us the result.

Now coming back to the main proof, by an integral form Taylor expansion of $V(x)\left(=G\left(d^{2} f(x)\right)\right)$ around $G\left(d^{2} f^{*}(x)\right)$, by the uniform boundedness of each element of second derivative $G$, one can get the following term bounded by $\sup _{x_{0}}\left\|d^{2} f(x)-d^{2} f^{*}(x)\right\|^{2}$ :

$$
\sup _{\substack{x_{0} \in \mathcal{G} \\ t \in\left[0, T_{x_{0}}^{*}\right]}}\left\|\int_{0}^{t}\left[\left(V-V^{*}\right)\left(\Upsilon_{x_{0}}^{*}(s)\right)-\nabla G\left(d^{2} f^{*}\left(\Upsilon_{x_{0}}^{*}(s)\right)\right) d^{2}\left(f-f^{*}\right)\left(\Upsilon_{x_{0}}^{*}(s)\right)\right] d s\right\| .
$$

In view of Lemma A.2, with the choice of $J$ in the assumption, $\sup _{x} \| d^{2} f(x)-$ $d^{2} f^{*}(x) \|^{2}$ is of order $(n / \log n)^{(5-\alpha) /(1+2 \alpha)}$. Now it suffices to prove that

$$
\sup _{\substack{x_{0} \in \mathcal{G} \\ t \in\left[0, T_{x_{0}}^{*}\right]}}\left\|\int_{0}^{t} \nabla G\left(d^{2} f^{*}\left(\Upsilon_{x_{0}}^{*}(s)\right)\right) d^{2}\left(f-f^{*}\right)\left(\Upsilon_{x_{0}}^{*}(s)\right) d s\right\|
$$


has posterior contraction rate $(n / \log n)^{(2-\alpha) /(1+2 \alpha)}$. To this end, we demonstrate the following quantity has this posterior contraction rate

$$
\sup _{\substack{x_{0} \in \mathcal{G} \\ t \in\left[0, T_{x_{0}}^{*}\right]}}\left|\int_{0}^{t} \frac{\partial G_{1}}{\partial x_{1}}\left(d^{2} f^{*}\left(\Upsilon_{x_{0}}^{*}(s)\right)\right)\left(f^{(2,0)}-f^{(2,0)^{*}}\right)\left(\Upsilon_{x_{0}}^{*}(s)\right) d s\right| .
$$

The rate for the other components can be derived similarly. Let $\mathcal{U}_{n}$ be a shrinking neighborhood of $\sigma_{0}^{2}$ such that $\hat{\sigma}_{n}^{2} \in \mathcal{U}_{n}$ and $\Pi\left(\sigma^{2} \in \mathcal{U}_{n} \mid \mathbb{D}_{n}\right) \rightarrow 1$ with probability tending to 1 .

Let $\tilde{G}\left(\Upsilon_{x_{0}}^{*}(s)\right)=\frac{\partial G_{1}}{\partial x_{1}}\left(d^{2} f^{*}\left(\Upsilon_{x_{0}}^{*}(s)\right)\right)$. To derive the rate, it suffices to show the rate for

$$
\mathrm{E}_{0}\left[\sup _{\sigma^{2} \in \mathcal{U}_{n}} \mathrm{E}\left(\sup _{x_{0}, t}\left|\int_{0}^{t} \tilde{G}\left(\Upsilon_{x_{0}}^{*}(s)\right)\left(f^{(2,0)}-f^{(2,0)^{*}}\right)\left(\Upsilon_{x_{0}}^{*}(s)\right) d s\right|^{2} \mid \mathbb{D}_{n}, \sigma^{2}\right)\right]
$$

decays as $(n / \log n)^{(4-2 \alpha) /(1+2 \alpha)}$, which then by Markov's inequality yields the desired posterior contraction rate. Notice that the posterior variance of $f^{(2,0)}(x)$ does not depend on $\mathbb{D}_{n}$, while its posterior mean $A_{(2,0)} Y+C_{(2,0)} \theta_{0}$ does not depend on $\sigma^{2}$. Also, in the posterior distribution conditional on $\sigma^{2}$, we have $f^{(2,0)}(x)-A_{(2,0)}(x) Y-C_{(2,0)}(x) \theta_{0}=\sigma b_{J_{1}, J_{2}}^{(2,0)}{ }^{T}(x)\left(B^{T} B+\Lambda_{0}^{-1}\right)^{-1 / 2} Z$, where $Z$ is a $J_{1} J_{2} \times 1$ vector of standard normal random variables. Thus we can upper bound the following

$$
\begin{aligned}
& \mathrm{E}_{0}\left[\sup _{\sigma^{2} \in \mathcal{U}_{n}} \mathrm{E}\left(\sup _{x_{0}, t}\left|\int_{0}^{t} \tilde{G}\left(\Upsilon_{x_{0}}^{*}(s)\right)\left(f^{(2,0)}-f^{(2,0)^{*}}\right)\left(\Upsilon_{x_{0}}^{*}(s)\right) d s\right|^{2} \mid \mathbb{D}_{n}, \sigma^{2}\right)\right] \\
& \lesssim \mathrm{E}_{0}\left[\sup _{\sigma^{2} \in \mathcal{U}_{n}} \mathrm{E}\left(\sup _{x_{0}, t}\left|\int_{0}^{t} \tilde{G}\left(\Upsilon_{x_{0}}^{*}(s)\right)\left(f^{(2,0)}-A_{(2,0)} Y-C_{(2,0)} \theta_{0}\right)\left(\Upsilon_{x_{0}}^{*}(s)\right) d s\right|^{2} \mid \sigma^{2}\right)\right] \\
& +\mathrm{E}_{0}\left[\sup _{x_{0}, t}\left|\int_{0}^{t} \tilde{G}\left(\Upsilon_{x_{0}}^{*}(s)\right)\left(A_{(2,0)} Y+C_{(2,0)} \theta_{0}-f^{(2,0)^{*}}\right)\left(\Upsilon_{x_{0}}^{*}(s)\right) d s\right|^{2}\right] \\
& \lesssim \sup _{\sigma^{2} \in \mathcal{U}_{n}} \sigma^{2} \mathrm{E}_{0}\left(\sup _{x_{0}, t}\left|\int_{0}^{t} \tilde{G}\left(\Upsilon_{x_{0}}^{*}(s)\right) b_{J_{1}, J_{2}}^{(2,0)}{ }^{T}\left(\Upsilon_{x_{0}}^{*}(s)\right) d s\left(B^{T} B+\Lambda_{0}^{-1}\right)^{-1 / 2} Z\right|^{2}\right) \\
& +\sup _{x_{0}, t} \mid \int_{0}^{t} \tilde{G}\left(\Upsilon_{x_{0}}^{*}(s)\right)\left(A_{(2,0)}\left(\Upsilon_{x_{0}}^{*}(s)\right) F^{*}+C_{(2,0)}\left(\Upsilon_{x_{0}}^{*}(s)\right) \theta_{0}-\left.f^{(2,0)^{*}}\left(\Upsilon_{x_{0}}^{*}(s)\right) d s\right|^{2}\right. \\
& +\mathrm{E}_{0}\left(\sup _{x_{0}, t}\left|\int_{0}^{t} \tilde{G}\left(\Upsilon_{x_{0}}^{*}(s)\right) A_{(2,0)}\left(\Upsilon_{x_{0}}^{*}(s)\right) \varepsilon d s\right|^{2}\right) .
\end{aligned}
$$

We shall tackle each of above three terms one by one. We let $a\left(x_{0}, t\right)=$ $\int_{0}^{t} \tilde{G}\left(\Upsilon_{x_{0}}^{*}(s)\right) b_{J_{1}, J_{2}}^{(2,0)}\left(\Upsilon_{x_{0}}^{*}(s)\right) d s$, where the integral is taken elementwise. Now consider the first term (A.2). Define $U_{1, n}\left(x_{0}, t\right)=a\left(x_{0}, t\right)\left(B^{T} B+\Lambda_{0}^{-1}\right)^{-1 / 2} Z$ which is Gaussian. For fixed $x_{0}, t$,

$$
\mathrm{E}\left[U_{1, n}\left(x_{0}, t\right)\right]^{2}=a\left(x_{0}, t\right)^{T}\left(B^{T} B+\Lambda_{0}^{-1}\right) a\left(x_{0}, t\right) \lesssim \frac{J^{2}}{n}\left\|a\left(x_{0}, t\right)\right\|^{2} \lesssim \frac{J^{5}}{n},
$$


where last step follows from Lemma A.5. Consider the two points $\left(x_{0}, t\right)$ and $\left(\tilde{x}_{0}, \tilde{t}\right)$, where the distance

$$
\begin{aligned}
d\left(\left(x_{0}, t\right),\left(\tilde{x}_{0}, \tilde{t}\right)\right) & :=\sqrt{\operatorname{Var}\left(U_{1, n}\left(x_{0}, t\right)-U_{1, n}\left(\tilde{x}_{0}, \tilde{t}\right)\right)} \\
& \lesssim\left(\left(a\left(x_{0}, t\right)-a\left(\tilde{x}_{0}, \tilde{t}\right)\right)^{T}\left(B^{T} B+\Lambda_{0}^{-1}\right)\left(a\left(x_{0}, t\right)-a\left(\tilde{x}_{0}, \tilde{t}\right)\right)\right)^{1 / 2} \\
& \lesssim\left(\frac{J^{2}}{n}\right)^{1 / 2}\left\|a\left(x_{0}, t\right)-a\left(\tilde{x}_{0}, \tilde{t}\right)\right\| \\
& \lesssim\left(\frac{J^{7}}{n}\left(|t-\tilde{t}|+\left\|x_{0}-\tilde{x}_{0}\right\|^{2}\right)\right)^{1 / 2} ;
\end{aligned}
$$

here the last line follows from Lemma A.5. Let $\tilde{d}\left(\left(x_{0}, t\right),\left(\tilde{x}_{0}, \tilde{t}\right)\right):=(|t-\tilde{t}|+$ $\left.\left\|x_{0}-\tilde{x}_{0}\right\|^{2}\right)^{1 / 2}$. Thus $d\left(\left(x_{0}, t\right),\left(\tilde{x}_{0}, \tilde{t}\right)\right) \lesssim \rho_{n} \tilde{d}\left(\left(x_{0}, t\right),\left(\tilde{x}_{0}, \tilde{t}\right)\right)$ for $\rho_{n}=J^{7 / 2} / n^{1 / 2}$. By Lemma A.11 of Yoo and Ghosal [38], setting $\delta_{n} \asymp 1 / J$ there, we obtain $\mathrm{E}\left(\sup _{x_{0}, t}\left[U_{1, n}\left(x_{0}, t\right)\right]^{2}\right)=O\left(J^{5} \log n / n\right)$. Therefore, (A.2) is of order $(\log n) J^{5} / n$.

For the term (A.4), let

$$
U_{2, n}\left(x_{0}, t\right)=\int_{0}^{t} \tilde{G}\left(\Upsilon_{x_{0}}^{*}(s)\right) A_{(2,0)}\left(\Upsilon_{x_{0}}^{*}(s)\right) \varepsilon d s=a\left(x_{0}, t\right)\left(B^{T} B+\Lambda_{0}^{-1}\right)^{-1} B^{T} \varepsilon
$$

Observe that for fix $x_{0}, t$,

$$
\begin{aligned}
\mathrm{E}\left[U_{2, n}\left(x_{0}, t\right)\right]^{2} & =\sigma_{0}^{2} a\left(x_{0}, t\right)^{T}\left(B^{T} B+\Lambda_{0}^{-1}\right)^{-1} B^{T} B\left(B^{T} B+\Lambda_{0}^{-1}\right)^{-1} a\left(x_{0}, t\right) \\
& \lesssim \sigma_{0}^{2} \frac{J^{2}}{n} \frac{n}{J^{2}} \frac{J^{2}}{n} a\left(x_{0}, t\right)^{T} a\left(x_{0}, t\right) \\
& \lesssim \sigma_{0}^{2} \frac{J^{5}}{n} .
\end{aligned}
$$

Since $\varepsilon$ is sub-Gaussian, $U_{2, n}\left(x_{0}, t\right)$ is sub-Gaussian with mean 0 and variance $\mathrm{E}\left[U_{2, n}\left(x_{0}, t\right)\right]^{2}$. By the same argument, $\mathrm{E}\left(\sup _{x_{0}, t}\left[U_{2, n}\left(x_{0}, t\right)\right]^{2}\right)=O\left(J^{5} \log n / n\right)$. Therefore, (A.4) is of order $(\log n) J^{5} / n$.

Finally, for the term (A.3), in view of Lemma A.1, the term

$$
\left|\int_{0}^{t} \tilde{G}\left(\Upsilon_{x_{0}}^{*}(s)\right)\left(A_{(2,0)}\left(\Upsilon_{x_{0}}^{*}(s)\right) F^{*}+C_{(2,0)}\left(\Upsilon_{x_{0}}^{*}(s)\right) \theta_{0}-f^{(2,0)^{*}}\left(\Upsilon_{x_{0}}^{*}(s)\right)\right) d s\right|
$$

can be bounded by

$$
\begin{aligned}
& \left|a\left(x_{0}, t\right)^{T}\left(B^{T} B+\Lambda_{0}^{-1}\right)^{-1}\left(B^{T}\left(F^{*}-B \theta_{\infty}\right)+\Lambda_{0}^{-1}\left(\theta_{0}-\theta_{\infty}\right)\right)\right| \\
+ & \left|\int_{0}^{t} \tilde{G}\left(\Upsilon_{x_{0}}^{*}(s)\right)\left(b_{J_{1}, J_{2}}^{(2,0)}{ }^{T}\left(\Upsilon_{x_{0}}^{*}(s)\right) \theta_{\infty}-f^{(2,0)^{*}}\left(\Upsilon_{x_{0}}^{*}(s)\right)\right) d s\right| .
\end{aligned}
$$

The first term of the above expression is bounded by

$$
\begin{aligned}
\| a\left(x_{0}, t\right) & \left\|_{1}\right\|\left(B^{T} B+\Lambda_{0}^{-1}\right)^{-1} \|_{(\infty, \infty)} \\
& \times\left(\left\|B^{T}\left(F^{*}-B \theta_{\infty}\right)\right\|_{\infty}+\left\|\Lambda_{0}^{-1}\right\|_{(\infty, \infty)}\left(\left\|\theta_{0}\right\|_{\infty}+\left\|\theta_{\infty}\right\|_{\infty}\right)\right) .
\end{aligned}
$$


Note that $\left\|a\left(x_{0}, t\right)\right\|_{1} \lesssim J^{2}$ by Lemma A.5. Since $\left\|\left(B^{T} B+\Lambda_{0}^{-1}\right)^{-1}\right\|_{(\infty, \infty)}=$ $O\left(J^{2} / n\right)$ and $\left\|B^{T}\left(F^{*}-B \theta_{\infty}\right)\right\|_{\infty}=O\left(n J^{-2-\alpha}\right)$, this term is of order $\left(J^{4} / n\right)+$ $J^{2-\alpha}$. The second term of the right hand side of above equation can be bounded by the supremum of approximate error which is of order $J^{2-\alpha}$. Therefore, (A.3) is of order $J^{4}\left(\left(J^{4} / n^{2}\right)+J^{-2 \alpha}\right)$.

Putting all the above terms together, (A.1) is of the order $(\log n / n) J^{5}+J^{4}\left(\left(J^{4} / n^{2}\right)+J^{-2 \alpha}\right)$. For $J \asymp(n / \log n)^{1 /(1+2 \alpha)}$, it becomes $(n / \log n)^{(4-2 \alpha) /(1+2 \alpha)}$, which then completes the proof.

Proof of Lemma A.5. We shall consider only two separate cases (i) $r=(2,0)$ and (ii) $r=(1,1)$.

(i). For $r=(2,0)$ (similarly for $r=(0,2)$ ),

$$
\begin{aligned}
\left\|a\left(x_{0}, t\right)\right\|_{1} & =\sum_{j_{1}=1}^{J_{1}} \sum_{j_{2}=1}^{J_{2}}\left|\int_{0}^{t} \tilde{G}_{1}(s) B_{j_{1}}^{\prime \prime}\left(\Upsilon_{1, x_{0}}^{*}(s)\right) B_{j_{2}}\left(\Upsilon_{2, x_{0}}^{*}(s)\right) d s\right| \\
& \lesssim \sum_{j_{1}=1}^{J_{1}} \sum_{j_{2}=1}^{J_{2}} \int_{0}^{t}\left|B_{j_{1}}^{\prime \prime}\left(\Upsilon_{1, x_{0}}^{*}(s)\right)\right| B_{j_{2}}\left(\Upsilon_{2, x_{0}}^{*}(s)\right) d s \\
& \leq \sum_{j_{1}}^{J_{1}} \int_{0}^{t}\left|B_{j_{1}}^{\prime \prime}\left(\Upsilon_{1, x_{0}}^{*}(s)\right)\right| d s,
\end{aligned}
$$

the last line follows by $\sum_{j_{2}=1}^{J_{2}} B_{j_{2}}\left(x_{2}\right)=1$ and using the fact that $B_{j_{1}, q_{1}}^{\prime \prime}(x)$ can be expressed as

$$
\begin{aligned}
& \frac{\left(q_{1}-1\right)\left(q_{1}-2\right) B_{j_{1}, q_{1}-2}(x)}{\left(t_{j_{1}}-t_{j_{1}-q_{1}+2}\right)\left(t_{j_{1}}-t_{j_{1}-q_{1}+1}\right)}+\frac{\left(q_{1}-1\right)\left(q_{1}-2\right) B_{j_{1}-1, q_{1}-2}(x)}{\left(t_{j_{1}-1}-t_{j_{1}-q_{1}+1}\right)\left(t_{j_{1}}-t_{j_{1}-q_{1}+1}\right)} \\
- & \frac{\left(q_{1}-1\right)\left(q_{1}-2\right) B_{j_{1}-1, q_{1}-2}(x)}{\left(t_{j_{1}-1}-t_{j_{1}-1-q_{1}+2}\right)\left(t_{j_{1}-1}-t_{j_{1}-q_{1}}\right)}+\frac{\left(q_{1}-1\right)\left(q_{1}-2\right) B_{j_{1}-2, q_{1}-2}(x)}{\left(t_{j_{1}-2}-t_{j_{1}-1-q_{1}+1}\right)\left(t_{j_{1}-1}-t_{j_{1}-q_{1}}\right)},
\end{aligned}
$$

which implies $\sum_{j_{1}=1}^{J_{1}}\left|B_{j_{1}}^{\prime \prime}\left(\Upsilon_{1, x_{0}}^{*}(s)\right)\right| \lesssim 4 J^{2}$. Therefore, $\left\|a\left(x_{0}, t\right)\right\|_{1} \lesssim J^{2}$.

Next, let $S_{j_{1}}=\left[t_{1, j_{1}-\left(q_{1}-2\right)}, t_{1, j_{1}}\right], S_{j_{2}}=\left[t_{2, j_{2}-q_{2}}, t_{2, j_{2}}\right]$ and $1_{j_{1}, j_{2}}(s):=\mathbb{1}\{s:$ $\left.\Upsilon_{x_{0}}^{*}(s) \in S_{j_{1}} \times S_{j_{2}}\right\}$. Turn to $\left\|a\left(x_{0}, t\right)\right\|^{2}$, which is

$$
\begin{aligned}
& \sum_{j_{1}=1}^{J_{1}} \sum_{j_{2}=1}^{J_{2}}\left(\int_{0}^{t} \tilde{G}_{1}(s) B_{j_{1}}^{\prime \prime}\left(\Upsilon_{1, x_{0}}^{*}(s)\right) B_{j_{2}}\left(\Upsilon_{2, x_{0}}^{*}(s)\right) d s\right)^{2} \\
= & \iint \tilde{G}_{1}(s) \tilde{G}_{1}\left(s^{\prime}\right) \\
\times & \sum_{j_{1}, j_{2}}\left(B_{j_{1}}^{\prime \prime}\left(\Upsilon_{1, x_{0}}^{*}(s)\right) B_{j_{2}}\left(\Upsilon_{2, x_{0}}^{*}(s)\right) B_{j_{1}}^{\prime \prime}\left(\Upsilon_{1, x_{0}}^{*}\left(s^{\prime}\right)\right) B_{j_{2}}\left(\Upsilon_{2, x_{0}}^{*}\left(s^{\prime}\right)\right)\right) d s d s^{\prime} \\
\lesssim & \iint \sum_{j_{1}, j_{2}}\left(\left|B_{j_{1}}^{\prime \prime}\left(\Upsilon_{1, x_{0}}^{*}(s)\right)\right| B_{j_{2}}\left(\Upsilon_{2, x_{0}}^{*}(s)\right)\left|B_{j_{1}}^{\prime \prime}\left(\Upsilon_{1, x_{0}}^{*}\left(s^{\prime}\right)\right)\right| B_{j_{2}}\left(\Upsilon_{2, x_{0}}^{*}\left(s^{\prime}\right)\right)\right) d s d s^{\prime}
\end{aligned}
$$




$$
\begin{aligned}
& =\iint \sum_{j_{1}, j_{2}} 1_{j_{1}, j_{2}}(s) 1_{j_{1}, j_{2}}\left(s^{\prime}\right) \\
& \times\left(\left|B_{j_{1}}^{\prime \prime}\left(\Upsilon_{1, x_{0}}^{*}(s)\right)\right| B_{j_{2}}\left(\Upsilon_{2, x_{0}}^{*}(s)\right)\left|B_{j_{1}}^{\prime \prime}\left(\Upsilon_{1, x_{0}}^{*}\left(s^{\prime}\right)\right)\right| B_{j_{2}}\left(\Upsilon_{2, x_{0}}^{*}\left(s^{\prime}\right)\right)\right) d s d s^{\prime} .
\end{aligned}
$$

The last equality is obtained as follows. Since for any fix $j_{1}, j_{2}, B_{j_{1}}^{\prime \prime}(\cdot)$ is supported on $S_{j_{1}}$ and $B_{j_{2}}(\cdot)$ is supported on $S_{j_{2}}$. So $1_{j_{1}, j_{2}}(s) 1_{j_{1}, j_{2}}\left(s^{\prime}\right)=\mathbb{1}\left\{\left(s, s^{\prime}\right)\right.$ : $\Upsilon_{x_{0}}^{*}(s) \in S_{j_{1}} \times S_{j_{2}}$ and $\left.\Upsilon_{x_{0}}^{*}\left(s^{\prime}\right) \in S_{j_{1}} \times S_{j_{2}}\right\}$. Notice that for $n$ large, $\left|S_{j_{1}}\right| \asymp$ $\left|S_{j_{2}}\right| \asymp J^{-1}$ and $\Upsilon_{x_{0}}^{*}(s) \in S_{j_{1}} \times S_{j_{2}}$ and $\Upsilon_{x_{0}}^{*}\left(s^{\prime}\right) \in S_{j_{1}} \times S_{j_{2}}$ implies that $\left\|\Upsilon_{x_{0}}^{*}(s)-\Upsilon_{x_{0}}^{*}\left(s^{\prime}\right)\right\| \lesssim J^{-1}$. By Assumption (A5), $C_{\mathcal{G}}\left|s-s^{\prime}\right| \leq \mid s-s^{\prime} \|\left(\Upsilon_{x_{0}}^{*}(s)-\right.$ $\left.\Upsilon_{x_{0}}^{*}\left(s^{\prime}\right)\right) /\left(s-s^{\prime}\right) \| \lesssim J^{-1}$, and hence $\left|s-s^{\prime}\right| \lesssim J^{-1}$. Therefore, for $n$ large enough, above quantity can be further bounded by a constant multiple of

$$
\begin{aligned}
& \iint \sum_{j_{1}, j_{2}} \mathbb{1}\left\{\left|s-s^{\prime}\right|<C J^{-1}\right\} \\
& \times\left(\left|B_{j_{1}}^{\prime \prime}\left(\Upsilon_{1, x_{0}}^{*}(s)\right)\right| B_{j_{2}}\left(\Upsilon_{2, x_{0}}^{*}(s)\right)\left|B_{j_{1}}^{\prime \prime}\left(\Upsilon_{1, x_{0}}^{*}\left(s^{\prime}\right)\right)\right| B_{j_{2}}\left(\Upsilon_{2, x_{0}}^{*}\left(s^{\prime}\right)\right)\right) d s d s^{\prime} .
\end{aligned}
$$

Noting $B_{j_{2}}\left(x_{2}\right) \leq 1$ and $\sum_{j_{2}=1}^{J_{2}} B_{j_{2}}=1$, it can be further bounded by

$$
\begin{aligned}
& \iint \sum_{j_{1}, j_{2}} \mathbb{1}\left\{\left|s-s^{\prime}\right|<C J^{-1}\right\}\left(\left|B_{j_{1}}^{\prime \prime}\left(\Upsilon_{1, x_{0}}^{*}(s)\right)\right| B_{j_{2}}\left(\Upsilon_{2, x_{0}}^{*}(s)\right)\left|B_{j_{1}}^{\prime \prime}\left(\Upsilon_{1, x_{0}}^{*}\left(s^{\prime}\right)\right)\right|\right) d s d s^{\prime} \\
& \lesssim \iint \sum_{j_{1}}^{J_{1}} \mathbb{1}\left\{\left|s-s^{\prime}\right|<C J^{-1}\right\}\left(\left|B_{j_{1}}^{\prime \prime}\left(\Upsilon_{1, x_{0}}^{*}(s)\right) B_{j_{1}}^{\prime \prime}\left(\Upsilon_{1, x_{0}}^{*}\left(s^{\prime}\right)\right)\right|\right) d s d s^{\prime} \\
& \lesssim J^{2} J^{-1} \int_{0}^{t} \sum_{j_{1}=1}^{J_{1}}\left|B_{j_{1}}^{\prime \prime}\left(\Upsilon_{1, x_{0}}^{*}(s)\right)\right| d s
\end{aligned}
$$

From argument used in bounding $\left\|a\left(x_{0}, t\right)\right\|_{1}$, we have $\sum_{j_{1}=1}^{J_{1}}\left|B_{j_{1}}^{\prime \prime}\left(\Upsilon_{1, x_{0}}^{*}(s)\right)\right| \lesssim$ $J^{2}$. This completes the proof for $\left\|a\left(x_{0}, t\right)\right\|^{2} \lesssim J^{3}$.

For the third result, we write

$$
\left\|a\left(x_{0}, t\right)-a\left(\tilde{x}_{0}, \tilde{t}\right)\right\|^{2} \lesssim\left\|a_{1}\left(x_{0}, t, \tilde{t}\right)\right\|^{2}+\left\|a_{2}\left(\tilde{t}, x_{0}, \tilde{x}_{0}\right)\right\|^{2},
$$

where

$$
a_{1}\left(x_{0}, t, \tilde{t}\right):=\int_{0}^{t} \tilde{G}\left(\Upsilon_{x_{0}}^{*}(s)\right) b_{J_{1}, J_{2}}^{(2,0)}\left(\Upsilon_{x_{0}}^{*}(s)\right) d s-\int_{0}^{\tilde{t}} \tilde{G}\left(\Upsilon_{x_{0}}^{*}(s)\right) b_{J_{1}, J_{2}}^{(2,0)}\left(\Upsilon_{x_{0}}^{*}(s)\right) d s,
$$

and

$$
a_{2}\left(\tilde{t}, x_{0}, \tilde{x}_{0}\right):=\int_{0}^{\tilde{t}} \tilde{G}\left(\Upsilon_{x_{0}}^{*}(s)\right) b_{J_{1}, J_{2}}^{(2,0)}\left(\Upsilon_{x_{0}}^{*}(s)\right) d s-\int_{0}^{\tilde{t}} \tilde{G}\left(\Upsilon_{\tilde{x}_{0}}^{*}(s)\right) b_{J_{1}, J_{2}}^{(2,0)}\left(\Upsilon_{\tilde{x}_{0}}^{*}(s)\right) d s .
$$

First, note that

$$
\left\|a_{1}\left(x_{0}, t, \tilde{t}\right)\right\|^{2}=\sum_{j_{1}=1}^{J_{1}} \sum_{j_{2}=1}^{J_{2}}\left(\int_{\tilde{t}}^{t} \tilde{G}\left(\Upsilon_{x_{0}}^{*}(s)\right) B_{j_{1}}^{\prime \prime}\left(\Upsilon_{1, x_{0}}^{*}(s)\right) B_{j_{2}}\left(\Upsilon_{2, x_{0}}^{*}(s)\right) d s\right)^{2}
$$




$$
\begin{aligned}
& \lesssim J^{2} J^{-1} \int_{t}^{\tilde{t}}\left(4 J^{2}\right) d s \\
& =J^{3}|t-\tilde{t}|,
\end{aligned}
$$

where the second line follows by a similar argument used to bound $\left\|a\left(x_{0}, t\right)\right\|^{2}$. Next, $\left\|a_{2}\left(\tilde{t}, x_{0}, \tilde{x}_{0}\right)\right\|^{2}$ is given by

$$
\begin{aligned}
\sum_{j_{1}=1}^{J_{1}} \sum_{j_{2}=1}^{J_{2}} & {\left[\int _ { 0 } ^ { \tilde { t } } \left(\tilde{G}\left(\Upsilon_{x_{0}}^{*}(s)\right) B_{j_{1}}^{\prime \prime}\left(\Upsilon_{1, x_{0}}^{*}(s)\right) B_{j_{2}}\left(\Upsilon_{2, x_{0}}^{*}(s)\right)\right.\right.} \\
& \left.\left.-\tilde{G}\left(\Upsilon_{\tilde{x}_{0}}^{*}(s)\right) B_{j_{1}}^{\prime \prime}\left(\Upsilon_{1, \tilde{x}_{0}}^{*}(s)\right) B_{j_{2}}\left(\Upsilon_{2, \tilde{x}_{0}}^{*}(s)\right) d s\right)\right]^{2}
\end{aligned}
$$

which is bounded (up to a constant multiple) by

$$
\begin{aligned}
& \sum_{j_{1}, j_{2}}\left(\int_{0}^{\tilde{t}} \tilde{G}\left(\Upsilon_{x_{0}}^{*}(s)\right)\left(B_{j_{1}}^{\prime \prime}\left(\Upsilon_{1, x_{0}}^{*}(s)\right)-B_{j_{1}}^{\prime \prime}\left(\Upsilon_{1, \tilde{x}_{0}}^{*}(s)\right)\right) B_{j_{2}}\left(\Upsilon_{2, x_{0}}^{*}(s)\right) d s\right)^{2} \\
& +\sum_{j_{1}, j_{2}}\left(\int_{0}^{\tilde{t}} \tilde{G}\left(\Upsilon_{x_{0}}^{*}(s)\right)\left(B_{j_{2}}\left(\Upsilon_{2, x_{0}}^{*}(s)\right)-B_{j_{2}}\left(\Upsilon_{2, \tilde{x}_{0}}^{*}(s)\right)\right) B_{j_{1}}^{\prime \prime}\left(\Upsilon_{1, \tilde{x}_{0}}^{*}(s)\right) d s\right)^{2} \\
& +\sum_{j_{1}, j_{2}}\left(\int_{0}^{\tilde{t}}\left(\tilde{G}\left(\Upsilon_{x_{0}}^{*}(s)\right)-\tilde{G}\left(\Upsilon_{\tilde{x}_{0}}^{*}(s)\right)\right) B_{j_{1}}^{\prime \prime}\left(\Upsilon_{1, \tilde{x}_{0}}^{*}(s)\right) B_{j_{2}}\left(\Upsilon_{2, \tilde{x}_{0}}^{*}(s)\right) d s\right)^{2} .
\end{aligned}
$$

Bound the first term in the right hand side of above expression as

$$
\begin{aligned}
& \sum_{j_{1}, j_{2}}\left(\int_{0}^{\tilde{t}} \tilde{G}\left(\Upsilon_{x_{0}}^{*}(s)\right)\left(B_{j_{1}}^{\prime \prime}\left(\Upsilon_{1, x_{0}}^{*}(s)\right)-B_{j_{1}}^{\prime \prime}\left(\Upsilon_{1, \tilde{x}_{0}}^{*}(s)\right)\right) B_{j_{2}}\left(\Upsilon_{2, x_{0}}^{*}(s)\right) d s\right)^{2} \\
\lesssim & \sum_{j_{1}, j_{2}}\left(\int_{0}^{\tilde{t}}\left|\tilde{G}\left(\Upsilon_{x_{0}}^{*}(s)\right)\left\|B_{j_{1}}^{\prime \prime \prime}\left(\Upsilon_{1, x_{0}}^{*}(s)\right)\right\| \Upsilon_{1, x_{0}}^{*}(s)-\Upsilon_{1, \tilde{x}_{0}}^{*}(s)\right| B_{j_{2}}\left(\Upsilon_{2, x_{0}}^{*}(s)\right) d s\right)^{2} \\
\lesssim & \left\|x_{0}-\tilde{x}_{0}\right\|^{2} \sum_{j_{1}, j_{2}}\left(\int_{0}^{\tilde{t}}\left|\tilde{G}\left(\Upsilon_{x_{0}}^{*}(s)\right)\right|\left|B_{j_{1}}^{\prime \prime \prime}\left(\Upsilon_{1, x_{0}}^{*}(s)\right)\right| B_{j_{2}}\left(\Upsilon_{2, x_{0}}^{*}(s)\right) d s\right)^{2} \\
\lesssim & \left\|x_{0}-\tilde{x}_{0}\right\|^{2} J^{2} \int_{0}^{\tilde{t}}\left|B_{j_{1}}^{\prime \prime \prime}\left(\Upsilon_{1, x_{0}}^{*}(s)\right)\right| d s \\
\lesssim & \left\|x_{0}-\tilde{x}_{0}\right\|^{2} J^{5} .
\end{aligned}
$$

The second term is bounded as

$$
\begin{aligned}
& \sum_{j_{1}=1}^{J_{1}} \sum_{j_{2}=1}^{J_{2}}\left(\int_{0}^{\tilde{t}} \tilde{G}\left(\Upsilon_{x_{0}}^{*}(s)\right)\left(B_{j_{2}}\left(\Upsilon_{2, x_{0}}^{*}(s)\right)-B_{j_{2}}\left(\Upsilon_{2, \tilde{x}_{0}}^{*}(s)\right)\right) B_{j_{1}}^{\prime \prime}\left(\Upsilon_{1, \tilde{x}_{0}}^{*}(s)\right) d s\right)^{2} \\
\lesssim & \sum_{j_{1}, j_{2}}\left(\int_{0}^{\tilde{t}}\left|\tilde{G}\left(\Upsilon_{x_{0}}^{*}(s)\right)\left\|\Upsilon_{2, x_{0}}^{*}(s)-\Upsilon_{2, \tilde{x}_{0}}^{*}(s)\right\| B_{j_{2}}^{\prime}\left(\Upsilon_{2, \tilde{x}_{0}}^{*}(s)\right) \| B_{j_{1}}^{\prime \prime}\left(\Upsilon_{1, \tilde{x}_{0}}^{*}(s)\right)\right|\right)^{2}
\end{aligned}
$$




$$
\begin{aligned}
& \lesssim\left\|x-\tilde{x}_{0}\right\|^{2} \sum_{j_{1}, j_{2}}\left(\int_{0}^{\tilde{t}}\left|\tilde{G}\left(\Upsilon_{x_{0}}^{*}(s)\right)\left\|B_{j_{2}}^{\prime}\left(\Upsilon_{2, \tilde{x}_{0}}^{*}(s)\right)\right\| B_{j_{1}}^{\prime \prime}\left(\Upsilon_{1, \tilde{x}_{0}}^{*}(s)\right)\right|\right)^{2} \\
& \lesssim\left\|x-\tilde{x}_{0}\right\|^{2} \int_{0}^{\tilde{t}} \mathbb{1}\left\{\left|s-s^{\prime}\right| \leq C J^{-1}\right\} \\
& \quad \times \sum_{j_{1}, j_{2}}\left|B_{j_{2}}^{\prime}\left(\Upsilon_{2, \tilde{x}_{0}}^{*}(s)\right)\left\|B_{j_{1}}^{\prime \prime}\left(\Upsilon_{1, \tilde{x}_{0}}^{*}(s)\right)\right\| B_{j_{2}}^{\prime}\left(\Upsilon_{2, \tilde{x}_{0}}^{*}\left(s^{\prime}\right)\right) \| B_{j_{1}}^{\prime \prime}\left(\Upsilon_{1, \tilde{x}_{0}}^{*}\left(s^{\prime}\right)\right)\right| d s d s^{\prime} \\
& \lesssim\left\|x-\tilde{x}_{0}\right\|^{2} \int_{0}^{\tilde{t}} \mathbb{1}\left\{\left|s-s^{\prime}\right| \leq C J^{-1}\right\} J^{2} \sum_{j_{1}=1}^{J_{1}}\left|B_{j_{1}}^{\prime \prime}\left(\Upsilon_{1, \tilde{x}_{0}}^{*}(s)\right) \| B_{j_{1}}^{\prime \prime}\left(\Upsilon_{1, \tilde{x}_{0}}^{*}\left(s^{\prime}\right)\right)\right| d s d s^{\prime} \\
& \lesssim J^{5}\left\|x_{0}-\tilde{x}_{0}\right\|^{2},
\end{aligned}
$$

where the second line follows from the mean value theorem, the third line from the Lipschitz continuity of $\Upsilon_{x_{0}}^{*}$ in $x_{0}$ (Remark A.1) whereas fourth line follows by a similar argument used to bound $\|a(x, t)\|^{2}$. The third term

$$
\begin{aligned}
\sum_{j_{1}=1}^{J_{1}} \sum_{j_{2}=1}^{J_{2}}\left(\int_{0}^{\tilde{t}}\left(\tilde{G}\left(\Upsilon_{x_{0}}^{*}(s)\right)-\tilde{G}\left(\Upsilon_{\tilde{x}_{0}}^{*}(s)\right)\right) B_{j_{1}}^{\prime \prime}\left(\Upsilon_{1, \tilde{x}_{0}}^{*}(s)\right) B_{j_{2}}\left(\Upsilon_{2, \tilde{x}_{0}}^{*}(s)\right) d s\right)^{2} \\
\quad \lesssim \sum_{j_{1}, j_{2}} \int_{0}^{\tilde{t}}\left(\left\|\Upsilon_{x_{0}}^{*}(s)-\Upsilon_{\tilde{x}_{0}}^{*}(s)\right\| B_{j_{1}}^{\prime \prime}\left(\Upsilon_{1, \tilde{x}_{0}}^{*}(s)\right) B_{j_{2}}\left(\Upsilon_{2, \tilde{x}_{0}}^{*}(s)\right) d s\right)^{2} \\
\quad \lesssim\left\|x_{0}-\tilde{x}_{0}\right\|^{2} \sum_{j_{1}, j_{2}}\left(\int_{0}^{\tilde{t}}\left|B_{j_{1}}^{\prime \prime}\left(\Upsilon_{1, \tilde{x}_{0}}^{*}(s)\right)\right| B_{j_{2}}\left(\Upsilon_{2, \tilde{x}_{0}}^{*}(s)\right) d s\right)^{2} \\
\quad \lesssim\left\|x_{0}-\tilde{x}_{0}\right\|^{2} J^{3}
\end{aligned}
$$

where the second line holds by mean value theorem and the third line holds due to Lipschitz continuity of $\Upsilon_{x_{0}}^{*}$ in $x_{0}$ (Remark A.1) and last line holds by similar argument for $\left\|a\left(x_{0}, t\right)\right\|^{2}$.

In summary, we have that $\left\|a_{2}\left(\tilde{t}, x_{0}, \tilde{x}_{0}\right)\right\|^{2} \lesssim J^{5}\left\|x_{0}-\tilde{x}_{0}\right\|^{2}$ and $\left\|a_{1}\left(x_{0}, t, \tilde{t}\right)\right\|^{2} \lesssim$ $J^{3}|t-\tilde{t}|$.

(ii). Now turning to the case $r=(1,1)$. By similar argument we have

$$
\begin{aligned}
\left\|a\left(x_{0}, t\right)\right\|_{1} & =\sum_{j_{1}=1}^{J_{1}} \sum_{j_{2}=1}^{J_{2}}\left|\int_{0}^{t} \tilde{G}_{1}(s) B_{j_{1}}^{\prime}\left(\Upsilon_{1, x_{0}}^{*}(s)\right) B_{j_{2}}^{\prime}\left(\Upsilon_{2, x_{0}}^{*}(s)\right) d s\right| \\
& \lesssim \sum_{j_{1}=1}^{J_{1}} \sum_{j_{2}=1}^{J_{2}} \int_{0}^{t}\left|B_{j_{1}}^{\prime}\left(\Upsilon_{1, x_{0}}^{*}(s)\right) \| B_{j_{2}}^{\prime}\left(\Upsilon_{2, x_{0}}^{*}(s)\right)\right| d s \\
& \leq J \sum_{j_{1}=1}^{J_{1}} \int_{0}^{t}\left|B_{j_{1}}^{\prime}\left(\Upsilon_{1, x_{0}}^{*}(s)\right)\right| d s \lesssim J^{2}
\end{aligned}
$$


Likewise, $\left\|a\left(x_{0}, t\right)\right\|^{2}=\sum_{j_{1}=1}^{J_{1}} \sum_{j_{2}=1}^{J_{2}}\left(\int_{0}^{t} \tilde{G}_{1}(s) B_{j_{1}}^{\prime}\left(\Upsilon_{1, x_{0}}^{*}(s)\right) B_{j_{2}}^{\prime}\left(\Upsilon_{2, x_{0}}^{*}(s)\right) d s\right)^{2}$ can be bounded by

$$
\begin{aligned}
\iint & \sum_{j_{1}=1}^{J_{1}} \sum_{j_{2}=1}^{J_{2}} \mathbb{1}\left\{\left|s-s^{\prime}\right|<C J^{-1}\right\} \\
& \times\left(\left|B_{j_{1}}^{\prime}\left(\Upsilon_{1, x_{0}}^{*}(s)\right) B_{j_{2}}^{\prime}\left(\Upsilon_{2, x_{0}}^{*}(s)\right) B_{j_{1}}^{\prime}\left(\Upsilon_{1, x_{0}}^{*}\left(s^{\prime}\right)\right) B_{j_{2}}^{\prime}\left(\Upsilon_{2, x_{0}}^{*}\left(s^{\prime}\right)\right)\right|\right) d s d s^{\prime}
\end{aligned}
$$

which can be further bounded as

$$
\begin{aligned}
& J \iint \sum_{j_{1}, j_{2}} \mathbb{1}\left\{\left|s-s^{\prime}\right|<C J^{-1}\right\} \\
& \quad \times\left(\left|B_{j_{1}}^{\prime}\left(\Upsilon_{1, x_{0}}^{*}(s)\right)\left\|B_{j_{2}}^{\prime}\left(\Upsilon_{2, x_{0}}^{*}(s)\right)\right\| B_{j_{1}}^{\prime}\left(\Upsilon_{1, x_{0}}^{*}\left(s^{\prime}\right)\right)\right|\right) d s d s^{\prime} \\
& \lesssim J^{2} \iint \sum_{j_{1}=1}^{J_{1}} \mathbb{1}\left\{\left|s-s^{\prime}\right|<C J^{-1}\right\}\left(\left|B_{j_{1}}^{\prime}\left(\Upsilon_{1, x_{0}}^{*}(s)\right) B_{j_{1}}^{\prime}\left(\Upsilon_{1, x_{0}}^{*}\left(s^{\prime}\right)\right)\right|\right) d s d s^{\prime} \\
& \lesssim J^{3} \iint \mathbb{1}\left\{\left|s-s^{\prime}\right|<C J^{-1}\right\} \sum_{j_{1}}^{J_{1}}\left(\left|B_{j_{1}}^{\prime}\left(\Upsilon_{1, x_{0}}^{*}(s)\right)\right|\right) d s d s^{\prime} \\
& \lesssim J^{3} J^{-1} \int_{0}^{t} \sum_{j_{1}=1}^{J_{1}}\left|B_{j_{1}}^{\prime}\left(\Upsilon_{1, x_{0}}^{*}(s)\right)\right| d s \\
& \lesssim J^{3} .
\end{aligned}
$$

The third result $\left\|a_{1}\left(x_{0}, t, \tilde{t}\right)\right\|^{2} \lesssim J^{3}|t-\tilde{t}|$ and $\left\|a_{2}\left(\tilde{t}, x, \tilde{x}_{0}\right)\right\|^{2} \lesssim J^{5}\left\|x_{0}-\tilde{x}_{0}\right\|^{2}$ can be derived in a similar manner, since

$$
\begin{aligned}
\left\|a_{1}\left(x_{0}, t, \tilde{t}\right)\right\|^{2} & =\sum_{j_{1}=1}^{J_{1}} \sum_{j_{2}=1}^{J_{2}}\left(\int_{\tilde{t}}^{t} \tilde{G}\left(\Upsilon_{x_{0}}^{*}(s)\right) B_{j_{1}}^{\prime}\left(\Upsilon_{1, x_{0}}^{*}(s)\right) B_{j_{2}}^{\prime}\left(\Upsilon_{2, x_{0}}^{*}(s)\right) d s\right)^{2} \\
& \lesssim J^{2} J^{-1} \int_{t}^{\tilde{t}}\left(4 J^{2}\right) d s \\
& =J^{3}|t-\tilde{t}|,
\end{aligned}
$$

where the second line follows by a similar argument used in bounding $\left\|a\left(x_{0}, t\right)\right\|^{2}$. Next, to bound $\left\|a_{2}\left(\tilde{t}, x_{0}, \tilde{x}_{0}\right)\right\|^{2}$, we need to estimate the following three terms

$$
\begin{aligned}
& \sum_{j_{1}=1}^{J_{1}} \sum_{j_{2}=1}^{J_{2}}\left(\int_{0}^{\tilde{t}} \tilde{G}\left(\Upsilon_{x_{0}}^{*}(s)\right)\left(B_{j_{1}}^{\prime}\left(\Upsilon_{1, x_{0}}^{*}(s)\right)-B_{j_{1}}^{\prime}\left(\Upsilon_{1, \tilde{x}_{0}}^{*}(s)\right)\right) B_{j_{2}}^{\prime}\left(\Upsilon_{2, x_{0}}^{*}(s)\right) d s\right)^{2}, \\
& \sum_{j_{1}=1}^{J_{1}} \sum_{j_{2}=1}^{J_{2}}\left(\int_{0}^{\tilde{t}} \tilde{G}\left(\Upsilon_{x_{0}}^{*}(s)\right)\left(B_{j_{2}}^{\prime}\left(\Upsilon_{2, x_{0}}^{*}(s)\right)-B_{j_{2}}^{\prime}\left(\Upsilon_{2, \tilde{x}_{0}}^{*}(s)\right)\right) B_{j_{1}}^{\prime}\left(\Upsilon_{1, \tilde{x}_{0}}^{*}(s)\right) d s\right)^{2}, \\
& \sum_{j_{1}=1}^{J_{1}} \sum_{j_{2}=1}^{J_{2}}\left(\int_{0}^{\tilde{t}}\left(\tilde{G}\left(\Upsilon_{x_{0}}^{*}(s)\right)-\tilde{G}\left(\Upsilon_{\tilde{x}_{0}}^{*}(s)\right)\right) B_{j_{1}}^{\prime}\left(\Upsilon_{1, \tilde{x}_{0}}^{*}(s)\right) B_{j_{2}}^{\prime}\left(\Upsilon_{2, \tilde{x}_{0}}^{*}(s)\right) d s\right)^{2} .
\end{aligned}
$$


This can be done by similar argument used for the case $r=(2,0)$ and we omit the details.

Proof of Proposition 5.2. We will first sketch a proof for that for any $\epsilon>0$ sufficiently small,

$$
\Pi\left(\sup _{x_{0} \in \mathcal{G}}\left|t_{x_{0}}^{*}-t_{x_{0}}\right|>\epsilon \mid \mathbb{D}_{n}\right) \stackrel{\mathrm{P}_{0}}{\longrightarrow} 0
$$

Recall that $t_{x_{0}}^{*}=\operatorname{argmin}\left\{|t| \geq 0:\left\langle\nabla f^{*}\left(\Upsilon_{x_{0}}^{*}(t)\right), V^{*}\left(\Upsilon_{x_{0}}^{*}(t)\right)\right\rangle=0, \lambda^{*}\left(\Upsilon_{x_{0}}^{*}(t)\right)<\right.$ $0\}$. Without loss of generality, we assume $t_{x_{0}}^{*}$ is nonnegative. Let $C_{x_{0}}=\{t \in$ $\left.\left[0, t_{x_{0}}^{*}-\epsilon\right]:\left\langle\nabla f^{*}\left(\Upsilon_{x_{0}}^{*}(t)\right), V^{*}\left(\Upsilon_{x_{0}}^{*}(t)\right)\right\rangle=0\right\}$. Note that it suffices to show the following set of assertions hold with posterior probabilities going to 1 ,

(1) $\inf _{x_{0} \in \mathcal{G}, t \in C_{x_{0}}} \lambda\left(\Upsilon_{x_{0}}(t)\right)>0$

(2) $\inf _{x_{0} \in \mathcal{G}, t \in\left[0, t_{x_{0}}^{*}-\epsilon\right] \backslash C_{x_{0}}}\left|\nabla f\left(\Upsilon_{x_{0}}(t)\right), V\left(\Upsilon_{x_{0}}(t)\right)\right\rangle \mid>0$,

(3) $\sup _{x_{0} \in \mathcal{G}, t \in\left[t_{x_{0}}^{*}-\epsilon, t_{x_{0}}^{*}+\epsilon\right]} \lambda\left(\Upsilon_{x_{0}}(t)\right)<0$,

(4) For all $x_{0} \in \mathcal{G}$, there exists $\tilde{t}_{x_{0}} \in\left[t_{x_{0}}^{*}-\epsilon, t_{x_{0}}^{*}+\epsilon\right]$ such that

$$
\left.\nabla f\left(\Upsilon_{x_{0}}\left(\tilde{t}_{x_{0}}\right)\right), V\left(\Upsilon_{x_{0}}\left(\tilde{t}_{x_{0}}\right)\right)\right\rangle=0
$$

When $C_{x_{0}}$ is empty, we can omit condition (1). By invoking the arguments similar to that in the proof of Proposition A.1 of Qiao and Polonik [30], with $\mathrm{P}_{0}$-probability tending to one, the above assertions hold with high posterior probability.

Now we are ready to establish the contraction rate. The proof of the bounds needed to establish this one is similar to that of Proposition 5.1 of Qiao and Polonik [30]. Let $D_{\Upsilon_{x_{0}}, V} f(t):=\nabla f\left(\Upsilon_{x_{0}}(t)\right)^{T} V\left(\Upsilon_{x_{0}}(t)\right)$ and

$$
\begin{aligned}
& D_{\Upsilon_{x_{0}}, V}^{2} f(t):=\left\langle\nabla\left\langle\nabla f\left(\Upsilon_{x_{0}}(t)\right), V\left(\Upsilon_{x_{0}}(t)\right)\right\rangle, V\left(\Upsilon_{x_{0}}(t)\right)\right\rangle \\
& =\nabla f\left(\Upsilon_{x_{0}}(t)\right)^{T} \nabla V\left(\Upsilon_{x_{0}}(t)\right) V\left(\Upsilon_{x_{0}}(t)\right)+V\left(\Upsilon_{x_{0}}(t)\right)^{T} H f\left(\Upsilon_{x_{0}}(t)\right) V\left(\Upsilon_{x_{0}}(t)\right)
\end{aligned}
$$

We have for some $\tilde{t}$ between $t_{x_{0}}$ and $t_{x_{0}}^{*}$,

$$
0=D_{\Upsilon_{x_{0}}, V} f\left(t_{x_{0}}\right)=D_{\Upsilon_{x_{0}}, V} f\left(t_{x_{0}}^{*}\right)+D_{\Upsilon_{x_{0}}, V}^{2} f(\tilde{t})\left(\tilde{t}-t_{x_{0}}^{*}\right)
$$

We claim that

$$
\Pi\left(\inf _{x_{0} \in \mathcal{G}}\left|D_{\Upsilon_{x_{0}}, V}^{2} f(\tilde{t})\right|>\eta \mid \mathbb{D}_{n}\right) \stackrel{\mathrm{P}_{0}}{\longrightarrow} 0
$$

Since

$$
\sup _{x_{0} \in \mathcal{G}}\left|D_{\Upsilon_{x_{0}}, V}^{2} f(\tilde{t})-D_{\Upsilon_{x_{0}}^{*}, V^{*}}^{2} f^{*}\left(t_{x_{0}}^{*}\right)\right| \geq\left|\inf _{x_{0} \in \mathcal{G}}\right| D_{\Upsilon_{x_{0}}, V}^{2} f(\tilde{t})\left|-\inf _{x_{0} \in \mathcal{G}}\right| D_{\Upsilon_{x_{0}}^{*}, V^{*}}^{2} f^{*}\left(t_{x_{0}}^{*}\right)||
$$

and by Assumption (A3), $\inf _{x_{0} \in \mathcal{G}}\left|D_{\Upsilon_{x_{0}}^{*}, V^{*}}^{2} f^{*}\left(t_{x_{0}}^{*}\right)\right| \geq \eta$, it suffices to show that with $\mathrm{P}_{0^{-}}$-probability tending to $1, \sup _{x \in \mathcal{G}}\left|D_{\Upsilon_{x_{0}}, V}^{2} f(\tilde{t})-D_{\Upsilon_{x_{0}}^{*}, V^{*}}^{2} f^{*}\left(t_{x_{0}}^{*}\right)\right|$ is small 
with high posterior probability. To see this, we write

$$
\begin{aligned}
\sup _{x_{0}}\left|D_{\Upsilon_{x_{0}}, V}^{2} f(\tilde{t})-D_{\Upsilon_{x_{0}}^{*}, V^{*}}^{2} f^{*}\left(t_{x_{0}}^{*}\right)\right| & \leq \sup _{x_{0}}^{2}\left|D_{\Upsilon_{x_{0}}, V}^{2} f(\tilde{t})-D_{\Upsilon_{x_{0}}^{*}, V^{*}}^{2} f^{*}(\tilde{t})\right| \\
& +\sup _{x_{0}}^{2}\left|D_{\Upsilon_{x_{0}}^{*}, V^{*}}^{2} f^{*}(\tilde{t})-D_{\Upsilon_{x_{0}}^{*}, V^{*}}^{2} f^{*}\left(t_{x_{0}}^{*}\right)\right| .
\end{aligned}
$$

The first term contracts to zero, simply due to the uniform contraction results for $\nabla f(x), V(x), \nabla V(x), H f(x)$ (Lemma A.2) and for $\Upsilon_{x_{0}}$ (Theorem 5.1). The second term contracts to zero, since $\Upsilon_{x_{0}}^{*}(t)$ is continuous in $t$ and $\nabla f^{*}(x), V^{*}(x)$, $\nabla V^{*}(x), H f^{*}(x)$ are uniform continuous. by

Also, $\sup _{x_{0}}\left|D_{\Upsilon_{x_{0}}, V} f\left(t_{x_{0}}^{*}\right)\right|=\sup _{x_{0}}\left|D_{\Upsilon_{x_{0}}, V} f\left(t_{x_{0}}^{*}\right)-D_{\Upsilon_{x_{0}}^{*}, V^{*}} f\left(t_{x_{0}}^{*}\right)\right|$ is given

$$
\begin{aligned}
& \sup _{x_{0}}\left|\left\langle\nabla f\left(\Upsilon_{x_{0}}\left(t_{x_{0}}^{*}\right)\right)-\nabla f^{*}\left(\Upsilon_{x_{0}}^{*}\left(t_{x_{0}}^{*}\right)\right), V\left(\Upsilon_{x_{0}}\left(t_{x_{0}}^{*}\right)\right)-V^{*}\left(\Upsilon_{x_{0}}^{*}\left(t_{x_{0}}^{*}\right)\right)\right\rangle\right| \\
+ & \sup _{x_{0}}\left|\left\langle\nabla f\left(\Upsilon_{x_{0}}\left(t_{x_{0}}^{*}\right)\right)-\nabla f^{*}\left(\Upsilon_{x_{0}}^{*}\left(t_{x_{0}}^{*}\right)\right), V^{*}\left(\Upsilon_{x_{0}}^{*}\left(t_{x_{0}}^{*}\right)\right)\right\rangle\right| \\
+ & \sup _{x_{0}}\left|\left\langle\nabla f^{*}\left(\Upsilon_{x_{0}}^{*}\left(t_{x_{0}}^{*}\right)\right), V\left(\Upsilon_{x_{0}}\left(t_{x_{0}}^{*}\right)\right)-V^{*}\left(\Upsilon_{x_{0}}^{*}\left(t_{x_{0}}^{*}\right)\right)\right\rangle\right| .
\end{aligned}
$$

Now since

$$
\sup _{x_{0}}\left|t_{x_{0}}-t_{x_{0}}^{*}\right| \leq \frac{1}{\inf _{x_{0}} D_{\Upsilon_{x_{0}}, V}^{2} f(\tilde{t})} \sup _{x_{0}}\left|D_{\Upsilon_{x_{0}}, V} f\left(t_{x_{0}}^{*}\right)\right|,
$$

we have

$$
\begin{aligned}
\sup _{x_{0} \in \mathcal{G}}\left|t_{x_{0}}^{*}-t_{x_{0}}\right| & \lesssim \sup _{x_{0} \in \mathcal{G}}\left(\left\|\nabla f\left(\Upsilon_{x_{0}}\left(t_{x_{0}}^{*}\right)\right)-\nabla f^{*}\left(\Upsilon_{x_{0}}^{*}\left(t_{x_{0}}^{*}\right)\right)\right\|\right) \sup _{x_{0} \in \mathcal{G}}\left\|V^{*}\left(\Upsilon_{x_{0}}^{*}\left(t_{x_{0}}^{*}\right)\right)\right\| \\
& +\sup _{x_{0} \in \mathcal{G}}\left(\left\|V\left(\Upsilon_{x_{0}}\left(t_{x_{0}}^{*}\right)\right)-V^{*}\left(\Upsilon_{x_{0}}^{*}\left(t_{x_{0}}^{*}\right)\right)\right\|\right) \sup _{x_{0} \in \mathcal{G}}\left\|\nabla f^{*}\left(\Upsilon_{x_{0}}^{*}\left(t_{x_{0}}^{*}\right)\right)\right\| .
\end{aligned}
$$

It is easy to see that

$$
\begin{aligned}
& \sup _{x_{0} \in \mathcal{G}} \| \nabla f\left(\Upsilon_{x_{0}}\left(t_{x_{0}}^{*}\right)\right)-\nabla f^{*}\left(\Upsilon_{x_{0}}^{*}\left(t_{x_{0}}^{*}\right)\right) \| \\
& \lesssim \sup _{x_{0} \in \mathcal{G}}\left\|\nabla f(x)-\nabla f^{*}(x)\right\|+\sup _{x_{0} \in \mathcal{G}} \sup _{t \in\left[0, T_{x_{0}}^{*}\right]}\left\|\Upsilon_{x_{0}}(t)-\Upsilon_{x_{0}}^{*}(t)\right\|, \\
& \sup _{x_{0} \in \mathcal{G}}\left\|V\left(\Upsilon_{x_{0}}\left(t_{x_{0}}^{*}\right)\right)-V^{*}\left(\Upsilon_{x_{0}}^{*}\left(t_{x_{0}}^{*}\right)\right)\right\| \\
& \quad \lesssim \sup _{x_{0} \in \mathcal{G}}\left\|V(x)-V^{*}(x)\right\|+\sup _{x_{0} \in \mathcal{G}} \sup _{t \in\left[0, T_{x_{0}}^{*}\right]}\left\|\Upsilon_{x_{0}}(t)-\Upsilon_{x_{0}}^{*}(t)\right\| .
\end{aligned}
$$

With the choice of $J \asymp(n / \log n)^{1 /(1+2 \alpha)}$, by Lemma A.2, $\sup _{x} \| \nabla f(x)-$ $\nabla f^{*}(x) \|$ has posterior contraction rate of order $(n / \log n)^{(3-2 \alpha) /(2(1+2 \alpha))}$, while $\sup _{x}\left\|V(x)-V^{*}(x)\right\|$ has that of order $(n / \log n)^{(5-2 \alpha) /(2(1+2 \alpha))}$. Therefore, considering the rate from Theorem 5.1, we have the desired result. 
Proof of Theorem 5.3. One can write

$$
\begin{aligned}
\Upsilon_{x_{0}}\left(t_{x_{0}}\right)-\Upsilon_{x_{0}}^{*}\left(t_{x_{0}}^{*}\right)= & \Upsilon_{x_{0}}\left(t_{x_{0}}\right)-\Upsilon_{x_{0}}\left(t_{x_{0}}^{*}\right)+\Upsilon_{x_{0}}\left(t_{x_{0}}^{*}\right)-\Upsilon_{x_{0}}^{*}\left(t_{x_{0}}^{*}\right) \\
= & V\left(\Upsilon_{x_{0}}\left(\tilde{t}_{x_{0}}\right)\right)\left(t_{x_{0}}-t_{x_{0}}^{*}\right)+\Upsilon_{x_{0}}\left(t_{x_{0}}^{*}\right)-\Upsilon_{x_{0}}^{*}\left(t_{x_{0}}^{*}\right) \\
= & V^{*}\left(\Upsilon_{x_{0}}^{*}\left(t_{x_{0}}^{*}\right)\right)\left(t_{x_{0}}-t_{x_{0}}^{*}\right)+\Upsilon_{x_{0}}\left(t_{x_{0}}^{*}\right)-\Upsilon_{x_{0}}^{*}\left(t_{x_{0}}^{*}\right) \\
& +\left(V\left(\Upsilon_{x_{0}}\left(\tilde{t}_{x_{0}}\right)\right)-V^{*}\left(\Upsilon_{x_{0}}^{*}\left(t_{x_{0}}^{*}\right)\right)\right)\left(t_{x_{0}}-t_{x_{0}}^{*}\right),
\end{aligned}
$$

for $\tilde{t}_{x_{0}}$ between $t_{x_{0}}$ and $t_{x_{0}}^{*}$. Therefore,

$$
\begin{aligned}
\sup _{x_{0} \in \mathcal{G}}\left\|\Upsilon_{x_{0}}\left(t_{x_{0}}\right)-\Upsilon_{x_{0}}^{*}\left(t_{x_{0}}^{*}\right)\right\| \lesssim \sup _{x_{0} \in \mathcal{G}}\left\|\Upsilon_{x_{0}}\left(t_{x_{0}}^{*}\right)-\Upsilon_{x_{0}}^{*}\left(t_{x_{0}}^{*}\right)\right\| \\
\quad+\sup _{x_{0} \in \mathcal{G}}\left|t_{x_{0}}-t_{x_{0}}^{*}\right|\left(1+\sup _{x_{0} \in \mathcal{G}}\left\|V\left(\Upsilon_{x_{0}}\left(\tilde{t}_{x_{0}}\right)\right)-V^{*}\left(\Upsilon_{x_{0}}^{*}\left(t_{x_{0}}^{*}\right)\right)\right\|\right) .
\end{aligned}
$$

In view of the posterior contraction of $V$ (Lemma A.2), Theorem 5.1 and Proposition 5.2, the posterior of $\sup _{x_{0} \in \mathcal{G}}\left\|V\left(\Upsilon_{x_{0}}\left(\tilde{t}_{x_{0}}\right)\right)-V^{*}\left(\Upsilon_{x_{0}}^{*}\left(t_{x_{0}}^{*}\right)\right)\right\|$ contracts to zero.

Since

$$
\sup _{x_{0} \in \mathcal{G}}\left\|\Upsilon_{x_{0}}\left(t_{x_{0}}\right)-\Upsilon_{x_{0}}^{*}\left(t_{x_{0}}^{*}\right)\right\| \lesssim \sup _{x_{0} \in \mathcal{G}}\left|t_{x_{0}}-t_{x_{0}}^{*}\right|+\sup _{x_{0} \in \mathcal{G}}\left\|\Upsilon_{x_{0}}\left(t_{x_{0}}^{*}\right)-\Upsilon_{x_{0}}^{*}\left(t_{x_{0}}^{*}\right)\right\|,
$$

by Theorem 5.1 and Proposition 5.2, we establish the posterior contraction rate for $\sup _{x_{0} \in \mathcal{G}}\left\|\Upsilon_{x_{0}}\left(t_{x_{0}}\right)-\Upsilon_{x_{0}}^{*}\left(t_{x_{0}}^{*}\right)\right\|$.

Proof of Proposition 5.6. Consider (A2) for (i). Note that

$$
\begin{aligned}
& \lambda\left(\Upsilon_{x_{0}}\left(t_{x_{0}}\right)\right)-\lambda^{*}\left(\Upsilon_{x_{0}}^{*}\left(t_{x_{0}}^{*}\right)\right) \\
& =V\left(\Upsilon_{x_{0}}\left(t_{x_{0}}\right)\right)^{T} H f\left(\Upsilon_{x_{0}}\left(t_{x_{0}}\right)\right) V\left(\Upsilon_{x_{0}}\left(t_{x_{0}}\right)\right) \\
& \quad-V^{*}\left(\Upsilon_{x_{0}}^{*}\left(t_{x_{0}}^{*}\right)\right)^{T} H f^{*}\left(\Upsilon_{x_{0}}^{*}\left(t_{x_{0}}^{*}\right)\right) V^{*}\left(\Upsilon_{x_{0}}^{*}\left(t_{x_{0}}^{*}\right)\right) .
\end{aligned}
$$

Thus by the posterior uniform contraction and uniform continuity of $V, H f$ (Lemma A.2) and the uniform contraction of $\Upsilon_{x_{0}}\left(t_{x_{0}}\right)$ (Theorem 5.3) over $x_{0}$, with $\mathrm{P}_{0}$-probability tending to 1 , clearly condition (A2) holds with high posterior probability. Condition (A3) trivially holds by the same uniform contraction results and noting that

$$
\begin{aligned}
& \left\langle\nabla\left\langle\nabla f\left(\Upsilon_{x_{0}}\left(t_{x_{0}}\right)\right), V\left(\Upsilon_{x_{0}}\left(t_{x_{0}}\right)\right)\right\rangle, V\left(\Upsilon_{x_{0}}\left(t_{x_{0}}\right)\right)\right\rangle \\
& =\nabla f\left(\Upsilon_{x_{0}}\left(t_{x_{0}}\right)\right)^{T} \nabla V\left(\Upsilon_{x_{0}}\left(t_{x_{0}}\right)\right) V\left(\Upsilon_{x_{0}}\left(t_{x_{0}}\right)\right) \\
& \quad+V\left(\Upsilon_{x_{0}}\left(t_{x_{0}}\right)\right)^{T} H f\left(\Upsilon_{x_{0}}\left(t_{x_{0}}\right)\right) V\left(\Upsilon_{x_{0}}\left(t_{x_{0}}\right)\right) .
\end{aligned}
$$

The compactness follows trivially by the continuity of $\lambda(x)$ and $\langle\nabla f(x), V(x)\rangle$ and the fact that intersection of closed sets is closed and the boundedness of $[0,1]^{2}$. Turning to Condition (A5): fix any $x_{0} \in \mathcal{G}$ and any $u, s$ such that $t_{x_{0}}^{*}$ 
$a^{*} \leq s<u \leq t_{x_{0}}^{*}+a^{*},\left\|\Upsilon_{x_{0}}(u)-\Upsilon_{x_{0}}(s)\right\| /(u-s)$ can be lower bounded as

$$
\begin{aligned}
& \frac{1}{u-s}\left\|\left(\Upsilon_{x_{0}}(u)-\Upsilon_{x_{0}}^{*}(u)\right)-\left(\Upsilon_{x_{0}}(s)-\Upsilon_{x_{0}}^{*}(s)\right)+\Upsilon_{x_{0}}^{*}(u)-\Upsilon_{x_{0}}^{*}(s)\right\| \\
& \geq \frac{1}{u-s}\left\|\Upsilon_{x_{0}}^{*}(u)-\Upsilon_{x_{0}}^{*}(s)\right\|-\frac{1}{u-s}\left\|\left(\Upsilon_{x_{0}}(u)-\Upsilon_{x_{0}}^{*}(u)\right)-\left(\Upsilon_{x_{0}}(s)-\Upsilon_{x_{0}}^{*}(s)\right)\right\|, \\
& \geq C_{\mathcal{G}}-\epsilon,
\end{aligned}
$$

for $\epsilon>0$ arbitrarily small, due to the contraction result in Theorem 5.1. Finally, in view of Lemma A.4 and Theorem 5.4, (ii) holds by similar argument.

Proof of Lemma 5.7. Let $x_{0}$ be an arbitrary point on $\hat{\mathcal{L}}$. Thus $\Upsilon_{x_{0}}(0)=x_{0} \in$ $\hat{\mathcal{L}}$. Suppose $\Upsilon_{x_{0}}\left(t_{x_{0}}\right) \in \mathcal{L}$ for some $t_{x_{0}} \neq 0$. Such $t_{x_{0}}$ exists in view of the assumptions of the lemma and the Remark A.1 (1). If $t_{x_{0}}=0$, then the upper bound holds trivially. Note that $\inf _{y \in \mathcal{L}}\left\|x_{0}-y\right\| \leq\left\|\Upsilon_{x_{0}}(0)-\Upsilon_{x_{0}}\left(t_{x_{0}}\right)\right\| \leq\left|t_{x_{0}}\right|$ as $\|V(x)\|=1$. Let $D \Upsilon_{\Upsilon_{0}, V} f(t):=\frac{d}{d t} f\left(\Upsilon_{x_{0}}(t)\right)=\nabla f\left(\Upsilon_{x_{0}}(t)\right)^{T} V\left(\Upsilon_{x_{0}}(t)\right)$ and

$$
\begin{aligned}
D_{\Upsilon_{x_{0}}, V}^{2} f(t):= & \left\langle\nabla\left\langle\nabla f\left(\Upsilon_{x_{0}}(t)\right), V\left(\Upsilon_{x_{0}}(t)\right)\right\rangle, V\left(\Upsilon_{x_{0}}(t)\right)\right\rangle \\
= & \nabla f\left(\Upsilon_{x_{0}}(t)\right)^{T} \nabla V\left(\Upsilon_{x_{0}}(t)\right) V\left(\Upsilon_{x_{0}}(t)\right) \\
& +V\left(\Upsilon_{x_{0}}(t)\right)^{T} H f\left(\Upsilon_{x_{0}}(t)\right) V\left(\Upsilon_{x_{0}}(t)\right),
\end{aligned}
$$

where the second equality is due to the chain rule. A Taylor expansion yields that

$$
D_{\Upsilon_{x_{0}}, V} f(t)-D_{\Upsilon_{x_{0}}, V} f\left(t_{x_{0}}\right)=\left(t-t_{x_{0}}\right) D_{\Upsilon_{x_{0}}, V}^{2} f(\tilde{t})
$$

for some $\tilde{t}$ between 0 and $t_{x_{0}}$. In particular, since $D_{\Upsilon_{x_{0}}, V} f\left(t_{x_{0}}\right)=0$, letting $t=0$, we obtain

$$
D_{\Upsilon_{x_{0}}, V} f(0)=-t_{x_{0}} D_{\Upsilon_{x_{0}}, V}^{2} f(\tilde{t})
$$

Furthermore,

$$
\begin{aligned}
\left|D_{\Upsilon_{x_{0}}, V} f(0)\right| & =\left|D_{\Upsilon_{x_{0}}, V} f(0)-D_{\Upsilon_{x_{0}}, \hat{V}} \hat{f}(0)\right| \\
& =\left|\nabla f\left(\Upsilon_{x_{0}}(0)\right)^{T} V\left(\Upsilon_{x_{0}}(0)\right)-\nabla \hat{f}\left(\Upsilon_{x_{0}}(0)\right)^{T} \hat{V}\left(\Upsilon_{x_{0}}(0)\right)\right| \\
& \leq \sup _{x}\left|\nabla f(x)^{T} V(x)-\nabla \hat{f}(x)^{T} \hat{V}(x)\right| \\
& \left.\leq \sup _{x}\left|\nabla f(x)^{T}(V(x)-\hat{V}(x))\right|+\sup _{x} \mid(\nabla f(x)-\nabla \hat{f}(x))^{T} \hat{V}(x)\right) \mid \\
& \left.\leq c_{0} \sup _{x}\|V(x)-\hat{V}(x)\|+\sup _{x}\|\nabla f(x)-\nabla \hat{f}(x)\|\right) \\
& \leq c_{0} \sup _{x}\|V(x)-\hat{V}(x)\|,
\end{aligned}
$$

where $c_{0}=\left(\sup _{x}\|\nabla f(x)\|\right) \vee 1$ (recalling $\|V\|=1$ ). By the uniform continuity of $\nabla f(x), \nabla V(x), V(x)$ and $H f(x)$ and the continuity of $\Upsilon_{x_{0}}(t)$ in $t$, without loss of generality, we can make $\left|t_{x_{0}}\right|$ small enough (see comments in the end of 
the proof). Thus we have

$$
\left|D_{\Upsilon_{x_{0}}, V}^{2} f(\tilde{t})-D_{\Upsilon_{x_{0}}, V}^{2} f\left(t_{x_{0}}\right)\right|<\eta / 2
$$

By Assumption (A3), $\left|D_{\Upsilon_{x_{0}, V}}^{2} f\left(t_{x_{0}}\right)\right|>\eta$, and hence

$$
\left|D_{\Upsilon_{x_{0}}, V}^{2} f(\tilde{t})\right| \geq\left|D_{\Upsilon_{x_{0}}, V}^{2} f\left(t_{x_{0}}\right)\right|-\left|D_{\Upsilon_{x_{0}}, V}^{2} f(\tilde{t})-D_{\Upsilon_{x_{0}}, V}^{2} f\left(t_{x_{0}}\right)\right|>\eta / 2 .
$$

Therefore, $\inf _{y \in \mathcal{L}}\left\|x_{0}-y\right\| \leq\left|t_{x_{0}}\right| \leq \frac{2 c_{0}}{\eta} \sup _{x}\|V(x)-\hat{V}(x)\|$. Thus $d(\hat{\mathcal{L}} \mid \mathcal{L}) \leq$ $\frac{2 c_{0}}{\eta} \sup _{x}\|V(x)-\hat{V}(x)\|$. Similarly, $d(\mathcal{L} \mid \hat{\mathcal{L}}) \leq \frac{2 \tilde{c}_{0}}{\eta} \sup _{x}\|V(x)-\hat{V}(x)\|$ for some fixed constant $\tilde{c}_{0}>0$. Therefore, $\operatorname{Haus}(\mathcal{L}, \hat{\mathcal{L}}) \leq \frac{c}{\eta} \sup _{x \in[0,1]^{2}}\|V(x)-\hat{V}(x)\|$ for some fixed constant $c>0$. In principle, we can use different $\eta$ in Assumption (A3) for $f$ and $\hat{f}$. For clarity, we use the same $\eta$. Allowing different constants do not change the final conclusion. Recall that $V(x)=G\left(d f^{2}(x)\right)$. Now since $G$ is a fixed Lipschitz continuous function, it is easy to get the upper bound for $\sup _{x \in[0,1]^{2}}\|V(x)-\hat{V}(x)\|$ in terms of the supremum distance of the derivatives of $f(x)-\hat{f}(x)$. Indeed, since

$$
\sqrt{\left(w_{1}-u_{1}\right)^{2}+4 v_{1}^{2}}-\sqrt{\left(w_{2}-u_{2}\right)^{2}+4 v_{2}^{2}} \leq\left|u_{1}-u_{2}\right|+\left|w_{1}-w_{2}\right|+2\left|v_{1}-v_{2}\right|,
$$

applying $c_{r}$-inequality, one can get

$$
\begin{aligned}
\sup _{x \in[0,1]^{2}}\|V(x)-\hat{V}(x)\| & \leq \sqrt{60} \sup _{x \in[0,1]^{2}}\left|f^{(2,0)}(x)-\hat{f}^{(2,0)}(x)\right| \\
& +\sqrt{60} \sup _{x \in[0,1]^{2}}\left|f^{(1,1)}(x)-\hat{f}^{(1,1)}(x)\right| \\
& +\sqrt{216} \sup _{x \in[0,1]^{2}}\left|f^{(0,2)}(x)-\hat{f}^{(0,2)}(x)\right| .
\end{aligned}
$$

At last, $\left|t_{x_{0}}\right|$ can be made arbitrarily small. To see this, if Assumption (A5) holds for the $f$ (or more precisely, $\Upsilon_{x_{0}}$ ), then $\left\|\Upsilon_{x_{0}}\left(t_{x_{0}}\right)-\hat{\Upsilon}_{x_{0}}\left(\hat{t}_{x_{0}}\right)\right\|=\| \Upsilon_{x_{0}}\left(t_{x_{0}}\right)-$ $x_{0}\|=\| \Upsilon_{x_{0}}\left(t_{x_{0}}\right)-\Upsilon_{x_{0}}(0) \|>C_{\mathcal{G}}\left|t_{x_{0}}\right|$. Since $\left\|\hat{\Upsilon}_{x_{0}}\left(\hat{t}_{x_{0}}\right)-\Upsilon_{x_{0}}\left(t_{x_{0}}\right)\right\|$ can be made arbitrarily small due to being close in supremum norm over $x_{0}$ (see previous theorems; for instance, Theorem 5.3, if here $\hat{f}$ is taken as $f$ from posterior samples, $f$ as the truth $\left.f^{*}\right),\left|t_{x_{0}}\right|$ can be made arbitrarily small. This completes the proof.

Proof of Theorem 5.9. For $\gamma<1 / 2$, by argument in the proof of Theorem of 5.3 of Yoo and Ghosal [38], one can establish that, for $r \in \mathcal{R}=\{(2,0),(1,1),(0,2)\}$,

$$
\begin{aligned}
& R_{n, r, \gamma} \asymp \mathrm{E}\left(\left\|f^{(r)}-\tilde{f}^{(r)}\right\|_{\infty} \mid \mathbb{D}_{n}\right), \\
& R_{n, r, \gamma}^{2} \asymp(\log n / n) J^{6} \asymp(\log n / n)^{(\alpha-2) /(\alpha+1)} .
\end{aligned}
$$

Recall from (3.2), $\Pi\left(f^{(r)} \mid \mathbb{D}_{n}\right) \sim \operatorname{GP}\left(\tilde{f}^{(r)}, \hat{\sigma}^{2} \Sigma_{r}\right)$. By Borell's inequality (see Proposition A.2.1 of Van der Vaart and Wellner [36]),

$$
\Pi\left(\mathcal{L} \notin C_{\mathcal{L}} \mid \mathbb{D}_{n}\right) \leq \Pi\left(\mathcal{L} \text { corresponds to } f \notin C_{f, r, \gamma}^{\rho} \text { for some } r \in \mathcal{R} \mid \mathbb{D}_{n}\right)
$$




$$
\begin{aligned}
& \leq \sum_{r \in \mathcal{R}} \Pi\left(\left\|f^{(r)}-\tilde{f}^{(r)}\right\|_{\infty}>\rho R_{n, r, \gamma} \mid \mathbb{D}_{n}\right) \\
& \leq 3 \max _{r \in \mathcal{R}} \exp \left[-c^{2} R_{n, r, \gamma}^{2} / c_{n, r}\right],
\end{aligned}
$$

for some constant $c>0$ and $c_{n, r}=\sup _{x} \operatorname{var}\left(f^{(r)}(x)-\tilde{f}^{(r)}(x) \mid \mathbb{D}_{n}\right)$, which is bounded by a constant multiple of $\sup _{x} \Sigma_{r}(x, x)$. But then it can be further bounded by

$$
\sup _{x}\left\|b_{J_{1}, J_{2}}^{(r)}(x)\right\|^{2}\left\|\left(B^{T} B+\Lambda_{0}^{-1}\right)^{-1}\right\|_{(2,2)} \lesssim(\log n)^{-3 /(\alpha+1)} n^{(2-\alpha) /(\alpha+1)} .
$$

Therefore, the above posterior probability tends to zero. Finally, by Lemma A.4 and noting that $R_{n, r, \gamma}$ is at least as big as $\delta_{n, 2, J}$ with high probability, $\mathrm{P}_{0}\left(\mathcal{L}^{*} \in C_{\mathcal{L}}\right)=\mathrm{P}_{0}\left(\left\|f^{*(r)}-\tilde{f}^{(r)}\right\|_{\infty} \leq \rho R_{n, r, \gamma}, \forall r \in \mathcal{R}\right) \rightarrow 1$, establishing the coverage of $C_{\mathcal{L}}$.

To see $C_{\mathcal{L}} \subset \bar{C}_{\mathcal{L}}$, for any $\mathcal{L} \in C_{\mathcal{L}}$, it is induced by some $f$ such that $\| f^{(r)}-$ $\tilde{f}^{(r)} \|_{\infty} \leq \rho \max _{r \in \mathcal{R}} R_{n, r, \gamma}$. In view of the discussion in the beginning of this section and Proposition 5.6, since Lemma 5.7 holds with $\mathrm{P}_{0}$-probability tending to 1 with $\mathcal{L}$ being the filament in posterior and $\hat{\mathcal{L}}$ being the filament induced by the posterior mean $\tilde{f}$, the result immediately follows.

\section{References}

[1] Arias-Castro, E., Donoho, D. L. and Huo, X. (2006). Adaptive multiscale detection of filamentary structures in a background of uniform random points, The Annals of Statistics 34(1): 326-349. MR2275244

[2] Belitser, E. (2017). On coverage and local radial rates of credible sets, The Annals of Statistics 45(3): 1124-1151. MR3662450

[3] Belitser, E. and Ghosal, S. (2018). Empirical Bayes oracle uncertainty quantification for regression, The Annals of Statistics (to appear), https://imstat.org/journals-and-publications/annals-of-statistics/ annals-of-statistics-future-papers/.

[4] Belitser, E. and Nurushev, N. (2015). Needles and straw in a haystack: robust confidence for possibly sparse sequences, arXiv preprint arXiv:1511.01803. MR4036032

[5] Castillo, I. (2014). On Bayesian supremum norm contraction rates, The Annals of Statistics 42(5): 2058-2091. MR3262477

[6] Chen, Y.-C., Genovese, C. R. and Wasserman, L. (2015). Asymptotic theory for density ridges, The Annals of Statistics 43(5): 1896-1928. MR3375871

[7] Chen, Y.-C., Ho, S., Brinkmann, J., Freeman, P. E., Genovese, C. R., Schneider, D. P. and Wasserman, L. (2015). Cosmic web reconstruction through density ridges: catalogue, Monthly Notices of the Royal Astronomical Society 454(1): 1140-1156.

[8] Chernozhukov, V., Chetverikov, D. and Kato, K. (2013). Gaussian approximations and multiplier bootstrap for maxima of sums of high-dimensional random vectors, The Annals of Statistics 41(6): 2786-2819. MR3161448 
[9] Chernozhukov, V., Chetverikov, D. and Kato, K. (2014). Anticoncentration and honest, adaptive confidence bands, The Annals of Statistics 42(5): 1140-1156. MR3262468

[10] Chernozhukov, V., Hong, H. and Tamer, E. (2007). Estimation and confidence regions for parameter sets in econometric models, Econometrica 75(5): 1243-1284. MR2347346

[11] Chernozhukov, V., Kocatulum, E. and Menzel, K. (2015). Inference on sets in finance, Quantitative Economics 6(2): 309-358. MR3388152

[12] Claeskens, G. and Van Keilegom, I. (2003). Bootstrap confidence bands for regression curves and their derivatives, The Annals of Statistics 31(6): 1852-1884. MR2036392

[13] De Boor, C. (1978). A Practical Guide to Splines, Springer-Verlag New York. MR0507062

[14] Dietrich, J. P., Werner, N., Clowe, D., Finoguenov, A., Kitching, T., Miller, L. and Simionescu, A. (2012). A filament of dark matter between two clusters of galaxies, Nature 487: 202-204.

[15] Eberly, D. (1996). Ridges in Image and Data Analysis, Kluwer, Boston, MA.

[16] Facer, M. R. and Müller, H.-G. (2003). Nonparametric estimation of the location of a maximum in a response surface, Journal of Multivariate Analysis 87(1): 191-217. MR2007268

[17] Genovese, C. R., Perone-Pacifico, M., Verdinelli, I. and Wasserman, L. (2012). Manifold estimation and singular deconvolution under Hausdorff loss, The Annals of Statistics 40(2): 941-963. MR2985939

[18] Genovese, C. R., Perone-Pacifico, M., Verdinelli, I. and Wasserman, L. (2014). Nonparametric ridge estimation, The Annals of Statistics 42(4): 1511-1545. MR3262459

[19] Giné, E. and Nickl, R. (2010). Confidence bands in density estimation, The Annals of Statistics 38(2): 1122-1170. MR2604707

[20] Giné, E. and Nickl, R. (2011). Rates of contraction for posterior distributions in $L^{r}$-metrics, $1 \leq r \leq \infty$, The Annals of Statistics 39(6): 2883-2911. MR3012395

[21] Jankowski, H. and Stanberry, L. (2012). Confidence regions in level set estimation, http://www.math.yorku.ca/ hkj/Research/level.pdf.

[22] Kim, Y.-H. (2009). Gronwall, bellman and pachpatte type integral inequalities with applications, Nonlinear Analysis: Theory, Methods $\&$ Applications 71(12): e2641-e2656. MR2672035

[23] Knapik, B. T., van der Vaart, A. W. and van Zanten, J. H. (2011). Bayesian inverse problems with Gaussian priors, The Annals of Statistics 39(5): 2626-2657. MR2906881

[24] Koltchinskii, V., Sakhanenko, L. and Cai, S. (2007). Integral curves of noisy vector fields and statistical problems in diffusion tensor imaging: Nonparametric kernel estimation and hypotheses testing, The Annals of Statistics 35(4): 1576-1607. MR2351098

[25] Mammen, E. and Polonik, W. (2013). Confidence regions for level sets, Journal of Multivariate Analysis 122: 202-214. MR3189318 
[26] Mason, D. M. and Polonik, W. (2009). Asymptotic normality of plug-in level set estimates, The Annals of Applied Probability 19(3): 1108-1142. MR2537201

[27] Molchanov, I. (2006). Theory of Random Sets, Springer Science \& Business Media. MR2132405

[28] Novikov, D., Colombi, S. and Doré, O. (2006). Skeleton as a probe of the cosmic web: the two-dimensional case, Monthly Notices of the Royal Astronomical Society 366(4): 1201-1216.

[29] Ozertem, U. and Erdogmus, D. (2011). Locally defined principal curves and surfaces, Journal of Machine Learning Research 12(4): 1249-1286. MR2804600

[30] Qiao, W. and Polonik, W. (2016). Theoretical analysis of nonparametric filament estimation, The Annals of Statistics 44(3): 1269-1297. MR3485960

[31] Ray, K. (2017). Adaptive Bernstein-von Mises theorems in Gaussian white noise, The Annals of Statistics 45(6): 2511-2536. MR3737900

[32] Schumaker, L. (2007). Spline Functions: Basic Theory, Cambridge University Press. MR2348176

[33] Shoung, J.-M. and Zhang, C.-H. (2001). Least squares estimators of the mode of a unimodal regression function, Annals of Statistics 29: 648-665. MR1865335

[34] Szabó, B., van der Vaart, A. and van Zanten, J. (2015). Frequentist coverage of adaptive nonparametric Bayesian credible sets, The Annals of Statistics 43(4): 1391-1428. MR3357861

[35] van der Pas, S., Szabó, B. and van der Vaart, A. (2017). Uncertainty quantification for the horseshoe (with discussion), Bayesian Analysis 12(4): 1221-1274. MR3724985

[36] Van der Vaart, A. W. and Wellner, J. A. (1996). Weak Convergence and Empirical Processes, Springer, New York. MR1385671

[37] Wasserman, L. (2016). Topological data analysis, https://arxiv.org/pdf/ 1609.08227.pdf.

[38] Yoo, W. W. and Ghosal, S. (2016). Supremum norm posterior contraction and credible sets for nonparametric multivariate regression, The Annals of Statistics 44(3): 1069-1102. MR3485954

[39] Yoo, W. W. and Ghosal, S. (2019). Bayesian mode and maximum estimation and accelerated rates of contraction, Bernoulli 25(3): 2330-2358. MR3961250 